\title{
Structural, electronic, and magnetic properties of a Mn monolayer on W(110)
}

\author{
M. Bode ${ }^{1, *}$ S. Heinze,${ }^{1,2 \dagger}$ A. Kubetzka, ${ }^{1}$ O. Pietzsch,${ }^{1}$ M. Hennefarth,${ }^{1}$ M. Getzlaff, ${ }^{1}$ R. Wiesendanger, ${ }^{1}$ X. Nie,${ }^{2}$ \\ G. Bihlmayer, ${ }^{2}$ and S. Blügel ${ }^{2, \sharp}$ \\ ${ }^{1}$ Institute of Applied Physics and Microstructure Research Center, University of Hamburg, Jungiusstrasse 11, \\ D-20355 Hamburg, Germany \\ ${ }^{2}$ Institut für Festkörperforschung, Forschungszentrum Jülich, D-52425 Jülich, Germany
}

(Received 30 August 2001; published 12 July 2002)

\begin{abstract}
In this paper we establish a monolayer of $\mathrm{Mn}$ on $\mathrm{W}(110)$ as a model system for two-dimensional itinerant antiferromagnetism. Combining scanning tunneling microscopy (STM), low-energy electron diffraction, and $a b$ initio calculations performed with the full-potential linearized augmented plane wave method we have studied the structural, electronic, and magnetic properties of a Mn monolayer on W(110). Our experimental results indicate that in spite of the huge tensile strain Mn grows pseudomorphically on W(110) up to a thickness of three monolayers. Intermixing between the Mn overlayer and the W substrate can be excluded. Using these structural data as a starting point for the ab initio calculations of one monolayer Mn on W(110) we conclude that (i) Mn is magnetic and exhibits a large magnetic moment of $3.32 \mu_{\mathrm{B}}$, (ii) the magnetic moments are arranged in a $c(2 \times 2)$ antiferromagnetic order, (iii) the easy axis of the magnetization is in plane and points along the $[1 \overline{1} 0]$ direction, i.e., the direction along the long side of the (110) surface unit cell with a magnetocrystalline anisotropy energy of $1.3-1.5 \mathrm{meV}$, and (iv) the $\mathrm{Mn}-\mathrm{W}$ interlayer distance is $2.14 \AA$. The calculated electronic structure of a Mn monolayer on W(110) is compared with experimental scanning tunneling spectroscopy results. Several aspects are in nice agreement, but one cannot unambiguously deduce the magnetic structure from such a comparison. The proposed two-dimensional antiferromagnetic ground state of a Mn monolayer on W(110) is directly verified by the use of spin-polarized STM (SP-STM) in the constant-current mode, and an in-plane easy magnetization axis could be confirmed using tips with different magnetization directions. We compare the measurements with theoretically determined SP-STM images calculated combining the Tersoff-Hamann model extended to SP-STM with the ab initio calculation, resulting in good agreement.

DOI: 10.1103/PhysRevB.66.014425

PACS number(s): 75.70.Ak, 68.37.Ef, 72.25.-b, 73.20.At
\end{abstract}

\section{INTRODUCTION}

Theoretically, the question of whether or not spontaneous magnetic order can occur under the condition of reduced dimensionality, i.e., in a one-dimensional (1D) linear chain or in a two-dimensional (2D) plane, has already been considered by Bloch ${ }^{1}$ in 1930 and-in a more rigorous work-by Mermin and Wagner in $1966 .{ }^{2}$ Both results were obtained within the isotropic Heisenberg model. It was found that neither long-range ferromagnetic (FM) nor antiferromagnetic (AFM) order is possible at finite temperature. For a very long time experimental contributions to the issue of thin film magnetism were hindered by numerous difficulties. On one hand, it was impossible up to now to prepare a freestanding 1D chain or a 2D film. However, significant progress was achieved by vapor deposition of magnetic materials on nonmagnetic substrates. Nowadays, the epitaxial growth of ultrathin, defect-free magnetic films on singlecrystalline substrates is a standard technique. ${ }^{3}$ On the other hand, one has to reach a sufficiently high sensitivity which allows one to measure the magnetic signal of, e.g., a film as thin as a single atomic layer. In the case of ultrathin ferromagnetic films several surface-specific and surface-adapted techniques have been developed ${ }^{3}$ such as, e.g., spin-polarized low-energy electron diffraction ${ }^{4}$ and the magneto-optical Kerr effect, ${ }^{5}$ respectively. Although it was claimed in some early publications ${ }^{6}$ that the first few monolayers (ML) are magnetically "dead" - probably an artifact of the electrolytic preparation-the existence of long-range ferromagnetic order for monolayer films is meanwhile indisputable from the theoretical point of view also. ${ }^{7}$

In contrast, only little experimental progress has been achieved in the case of antiferromagnetic films. This lack of experimental work is even more annoying as the rapid development in computational physics allows one to calculate the magnetic properties of specific and realistic overlayersubstrate systems with high accuracy. In particular, more than ten years ago Blügel et al. already gave detailed predictions for the existence of $2 \mathrm{D}$ antiferromagnetic monolayer films of V, Cr, and Mn on (100) oriented Pd substrates ${ }^{8}$ and later also on noble-metal substrates. ${ }^{9}$ In such a film the magnetic moments of nearest-neighbor atoms couple antiferromagnetically to each other, giving rise to a checkerboard arrangement of magnetic moments with opposite orientation. From these $a b$ initio calculations based upon the fullpotential linearized augmented plane wave (FLAPW) method, it was concluded that the hybridization between the monolayer and the noble-metal substrate is negligible and the monolayer film behaves as a perfect 2D system.

There have been several attempts to verify the existence of these 2D antiferromagnets and some evidence has been given. ${ }^{10-13}$ However, definite experimental proof was hindered by three problems: (i) First, the preparation of flat $3 d$-metal overlayers on noble-metal substrates with an atomically sharp interface is very demanding. Interdiffusion between the substrate and the overlayer has been predicted theoretically ${ }^{14}$ as well as observed experimentally even at room temperature. ${ }^{15}$ This problem is caused by a lower sur- 
face free energy of noble metals than of $3 d$ metals. ${ }^{16}$ (ii) Second, the Néel temperature below which magnetic order exists is unknown and probably very low. (iii) The main problem, however, is related to the AFM structure itself: due to the fact that in this ultimate limit adjacent atoms at nearest-neighbor sites have magnetic moments with opposite directions the total (average) magnetization cancels on length scales beyond the atomic scale. Therefore, spatially averaging techniques like those mentioned above are not suitable to decide whether or not a thin film is in a $2 \mathrm{D}$ antiferromagnetic ground state.

The problem of interdiffusion can be avoided by choosing a more stable substrate like $\mathrm{W}(110)$ which has become very popular for the growth of thin magnetic films, as, such e.g., $\mathrm{Fe}$ on $\mathrm{W}(110) .{ }^{17}$ Indeed, a recent scanning tunneling microscopy (STM) study revealed that up to a local coverage of three atomic layers $\mathrm{Mn}$ grows pseudomorphically on $\mathrm{W}(110) .{ }^{18}$ The drawback is the strong hybridization of the $3 d$ monolayer with such a $5 d$ substrate, influencing the magnetic properties. As a consequence, the $3 d$ bands of the transition-metal broaden. This may reduce the exchange splitting and thereby also the magnetic moment. Nevertheless, the two-dimensional antiferromagnetism within the monolayer film should not be destroyed since it does not depend on a high density of states at the Fermi energy, as in the case of ferromagnetism, but rather on a low density of states in the center of the $d$ band which sometimes becomes even lower due to the hybridization with the substrate. The second problem, i.e., the unknown and probably very low Néel temperature, can be solved by cooling the sample with liquid helium. Problem (iii), however, requires a measurement technique which combines magnetic sensitivity with high spatial resolution on an atomic scale. Obviously, these requirements are fulfilled for spin-polarized scanning tunneling spectroscopy (SP-STM). ${ }^{19,20}$

In a recent publication ${ }^{21}$ we have shown that lowtemperature SP-STM enables the imaging of the antiferromagnetic superstructure of the Mn monolayer. In this article we present a survey of our experimental and theoretical work, which was conducted in order to investigate the structural, electronic, and magnetic properties of a single $\mathrm{Mn}$ monolayer on W(110). Details of the computational and experimental procedure as well as a description of the applied model of SP-STM are given in Sec. II. We will show with STM and LEED data that in spite of the large misfit between adlayer and substrate and the resulting tensile strain $\mathrm{Mn}$ grows pseudomorphically on bcc $\mathrm{W}(110)$, i.e., in the $\delta$ phase (Sec. III A). On the basis of this structure determination we have calculated the interlayer spacing, the magnetic ground state, and the easy magnetization axis by means of the FLAPW method (Sec. III B). The band structure of the Mn monolayer on $\mathrm{W}(110)$ is compared with experimental scanning tunneling spectroscopy (STS) results in Sec. III C. Finally, in Sec. III D we present general arguments as well as explicit calculations demonstrating that the use of a magnetic STM probe tip allows the direct imaging of surface magnetic superstructures. In particular, experimental data verifying this prediction are presented for the $\mathrm{Mn}$ monolayer on $\mathrm{W}(110)$. By a thorough analysis of the calculation we can unambiguously relate the magnetic contrast obtained to a specific surface state.

\section{DETAILS OF REALIZATION}

\section{A. Computational details}

$A b$ initio calculations of the electronic structure of one monolayer of Mn on W(110) have been carried out on the basis of density functional theory. As the local spin-density approximation $^{22}$ (LSDA) to the exchange-correlation potential has been shown to systematically underestimate lattice constants and thereby also the interlayer distances of $3 d$ transition metals, we have applied the generalized gradient approximation (GGA) of Perdew and Wang. ${ }^{23}$ For the optimized GGA equilibrium structure, properties of the electronic structure such as the magnetic moment, the magnetocrystalline anisotropy energy, or the local density of states differ only little with the particular exchange-correlation potential used and can be calculated by either the GGA or the LSDA, e.g., of Moruzzi, Janak, and Williams. ${ }^{24}$

We have employed the full-potential linearized augmented plane wave method in bulk and film geometry ${ }^{25,26}$ as implemented in the FLEUR code. ${ }^{27}$ The pseudomorphic Mn film on W(110) was modeled by a symmetric slab consisting of nine layers of bcc W(110) substrate, a Mn monolayer on both sides of the film, and a semi-infinite vacuum region on both sides of the monolayer. The calculations of the interlayer relaxation of the different magnetic structures were carried out in two-dimensional $c(2 \times 2)$ and $p(2 \times 2)$ unit cells with two atoms and four atoms per layer, respectively. For the relaxed interlayer distances the energy differences between the different magnetic structures were compared in the $p(2 \times 2)$ unit cell. For $\mathrm{W}$, we assumed the experimental lattice constant of $a_{\mathrm{W}}=3.165 \AA$ and the ideal bcc interlayer spacing, as the $\mathrm{W}$ interlayer relaxation is small as shown by Qian and Hübner ${ }^{28}$ for $\mathrm{Fe}$ on W(110). Due to the large difference in the (in-plane) lattice constants between Mn and W, pseudomorphic growth conditions introduce a large strain on $\mathrm{Mn}$, and strong Mn interlayer relaxations are expected. Since the magnetic moments and the equilibrium magnetic structure may depend strongly on these structural details, we determined the equilibrium interlayer distance between the $\mathrm{Mn}$ monolayer and the $\mathrm{W}(110)$ surface for different magnetic structures by employing total-energy calculations. All displayed results refer to the equilibrium interlayer distances.

In an earlier publication (Ref. 21) we determined the equilibrium structure by the LSDA method. A comparison of these results with the improved ones obtained by the GGA shows that absolute numbers concerning, for example, the energy differences between the magnetic configurations and the values of the optimized interlayer distances change, but all conclusions concerning the magnetic structure, the easy magnetization axis, and the analysis of spin-polarized STM measurements remain unaffected. In particular, it is expected that the last remains unaffected since the topography of the SP-STM images is based on more general arguments. ${ }^{29}$

The basis set used for the valence states consisted of about 80 augmented plane waves per atom in the unit cell. The W $5 p$ and the Mn $3 p$ states were treated as core states. ${ }^{30}$ 
Nonspherical terms in the potential, charge density, and wave functions were expanded within the muffin-tin spheres with radii $R_{\mathrm{MT}}^{\mathrm{W}}=2.456$ a.u. $(1$ a.u. $=0.5292 \AA)$ and $R_{\mathrm{MT}}^{\mathrm{Mn}}$ $=2.1$ a.u., up to $l_{\max } \leqslant 8$. The self-consistent electronic structure was determined with $35 \mathbf{k}_{\|}$points in the irreducible part (1/4) of the two-dimensional Brillouin zone (I2BZ). The integrated local density of states (ILDOS) resolved over the 2DBZ was analyzed using $176 \mathbf{k}_{\|}$points in the I2BZ. The star coefficients and from these also the STM images and corrugation amplitudes were calculated on this $\mathbf{k}_{\|}$-point set with a Gaussian broadening of $25 \mathrm{meV}$. A variation of the broadening leads merely to insignificant quantitative changes.

To determine the magnetocrystalline anisotropy energy and the easy axis of the magnetization, we carried out calculations including the spin-orbit coupling in a second variational procedure. ${ }^{31}$ The total-energy calculations of the magnetocrystalline anisotropy energy were performed with $783 \mathbf{k}_{\|}$points in the magnetic I2BZ (1/2 of the BZ). All occupied and empty states up to $1.2 \mathrm{Ry}$ above the Fermi energy were included as a second variational basis set to solve the relativistic eigenvalue problem. In these calculations we used films with a W thickness of $5 \mathrm{ML}$. This is sufficient to guarantee a stable magnetocrystalline anisotropy energy value to within a few percent.

\section{B. Theoretical model of spin-polarized STM}

In the general case of a spin-polarized STM measurement the tunneling current $I$ that gives rise to the STM images is a function of the applied bias voltage $U$ and in an extension of the Tersoff-Hamann model $^{32}$ is given by ${ }^{29}$

$$
\begin{aligned}
I\left(\mathbf{r}_{\|}, z, U, \theta\right) \propto & \int_{-\infty}^{+\infty} g_{U, T}(\epsilon)\left[n\left(\mathbf{r}_{\|}, z, \epsilon\right)\right. \\
& \left.+P_{T} \cos \theta m\left(\mathbf{r}_{\|}, z, \epsilon\right)\right] d \epsilon .
\end{aligned}
$$

$P_{T}$ denotes the spin polarization of the tip. $n\left(\mathbf{r}_{\|}, z, \epsilon\right)$ and $m\left(\mathbf{r}_{\|}, z, \boldsymbol{\epsilon}\right)$ are the (spin-summed) local density of states (LDOS) and the local spin density of states (LSDOS) of the sample, respectively, evaluated at the lateral $\left(\mathbf{r}_{\|}\right)$and vertical (z) position of the tip. ${ }^{33} \theta$ is the angle between the magnetization axes of the tip $\mathbf{M}_{T}$ and the sample $\mathbf{M}_{S} \cdot{ }^{34} g_{U, T}$ is the difference of the Fermi function $f_{T}$ at $\epsilon_{F}-e U+\epsilon$ and $\epsilon_{F}$ $+\epsilon$. The LDOS and the LSDOS are related in a trivial manner to the local density of states for the majority $(\uparrow)$ and minority $(\downarrow)$ electrons:

$$
\begin{aligned}
& n\left(\mathbf{r}_{\|}, z, \boldsymbol{\epsilon}\right)=n_{\uparrow}\left(\mathbf{r}_{\|}, z, \epsilon\right)+n_{\downarrow}\left(\mathbf{r}_{\|}, z, \epsilon\right), \\
& m\left(\mathbf{r}_{\|}, z, \epsilon\right)=n_{\uparrow}\left(\mathbf{r}_{\|}, z, \epsilon\right)-n_{\downarrow}\left(\mathbf{r}_{\|}, z, \epsilon\right) .
\end{aligned}
$$

For a periodic surface the LDOS's for majority and minority electrons, $n_{\uparrow}\left(\mathbf{r}_{\|}, z, \boldsymbol{\epsilon}\right)$ and $n_{\downarrow}\left(\mathbf{r}_{\|}, z, \boldsymbol{\epsilon}\right)$, and thereby also $n\left(\mathbf{r}_{\|}, z, \boldsymbol{\epsilon}\right)$ and $m\left(\mathbf{r}_{\|}, z, \boldsymbol{\epsilon}\right)$, can be expanded in so-called star functions $\phi_{s}$ (see also Ref. 35), which are symmetrized 2D plane waves (a sum of plane waves with reciprocal lattice vectors related by symmetry operations of the $2 \mathrm{D}$ point group):

$$
n_{\sigma}\left(\mathbf{r}_{\|}, z, \epsilon\right)=\sum_{s} n_{\sigma}^{s}(z, \epsilon) \phi_{s}\left(\mathbf{r}_{\|}\right)
$$

where $\sigma$ denotes the spin and can be of value $(\uparrow, \downarrow)$. The expansion coefficients $n_{\sigma}^{s}(z, \epsilon)$ are distance and energy dependent, and in the following we will call them star coefficients. The star functions are numbered by the order of the length of the representative reciprocal lattice vector $\mathbf{G}_{\|}^{s}$. For $s=0$ we find $\mathbf{G}_{\|}^{0}=0$ and the star function $\phi_{0}$ is simply a constant. Thus it does not contribute to the corrugation pattern. Its coefficient $n_{\sigma}^{0}$ must be positive since it represents the charge integrated over the 2D unit cell. The higher star functions $s>0$ are responsible for the STM image. The star coefficients $n_{\sigma}^{s}$ decay exponentially with increasing distance $z$ and increasing length $\left|\mathbf{G}_{\|}^{S}\right|$ of the reciprocal lattice vector. ${ }^{35}$

$$
\begin{aligned}
n_{\sigma}^{s}(z, \boldsymbol{\epsilon}) \approx & n_{\sigma}^{s}(\boldsymbol{\epsilon}) \exp \left[-z \sqrt{2 m|\epsilon| / \hbar^{2}+\left(\mathbf{k}_{\|}^{\min }\right)^{2}}\right] \\
& \times \exp \left[-z \sqrt{2 m|\epsilon| / \hbar^{2}+\left(\mathbf{k}_{\|}^{\min }+\mathbf{G}_{\|}^{s}\right)^{2}}\right] \\
\approx & n_{\sigma}^{s}(\boldsymbol{\epsilon}) \exp \left[-2 z \sqrt{2 m|\epsilon| / \hbar^{2}+\left(\mathbf{G}_{\|}^{s} / 2\right)^{2}}\right],
\end{aligned}
$$

where $\epsilon_{F}$ is the Fermi energy and $\mathbf{k}_{\|}^{\min }$ is the wave vector within the two-dimensional Brillouin zone that minimizes the term $\left(\mathbf{k}_{\|}+\mathbf{G}_{\|}^{s}\right)^{2}$. The second line applies if $\mathbf{G}_{\|}^{s} / 2$ is within the first Brillouin zone. The exponential decay with increasing length of $\mathbf{G}_{\|}^{s}$ allows one to neglect higher-order star functions. Thus the laterally nonconstant part of the LDOS (which is equivalent to the STM image) is dominated by the star function with the lowest nonvanishing $\mathbf{G}_{\|}^{s}$ vector. The corrugation amplitude $\Delta z$, i.e., the maximum variation in the vertical position of the tip as it scans the surface at constant current, can be calculated as described in Ref. 35 .

Within the described theoretical treatment any variation in the tip DOS is neglected. This approximation is justified at small bias voltages, for tips with a flat DOS, or if the variation of the sample DOS is more pronounced. Although the two latter requirements are not strictly fulfilled, ${ }^{36,37}$ this model of the tunneling current combined with ab initio calculations of sample properties yields good agreement with experimental STM data for metal surfaces ${ }^{38-40}$ as well as bias-voltage-dependent STM images of surface alloys. ${ }^{41,42}$

\section{Experimental setup and procedures}

The experiments were performed in an ultrahigh-vacuum (UHV) system with separate chambers for substrate preparation, sample transfer, metal vapor deposition, surface analysis, and cryogenic STM, allowing the preparation and characterization of the sample surface without any exposure to an ambient atmosphere. ${ }^{43}$ The base pressure in each chamber is in the low $10^{-11}$ torr range.

The W(110) crystals are mounted via tungsten wires (thickness $0.4-0.5 \mathrm{~mm}$ ) on a tungsten plate with a thickness of $1 \mathrm{~mm}$. This sample stack is prepared by numerous cycles of long-term heating at $1500 \mathrm{~K}$ in an oxygen atmosphere of $10^{-7}-10^{-6}$ torr and subsequent flashing up to $2500 \mathrm{~K}^{44}$ After this preparation both crystals gave a sharp $(1 \times 1)$ lowenergy electron diffraction (LEED) pattern. No traces of con- 


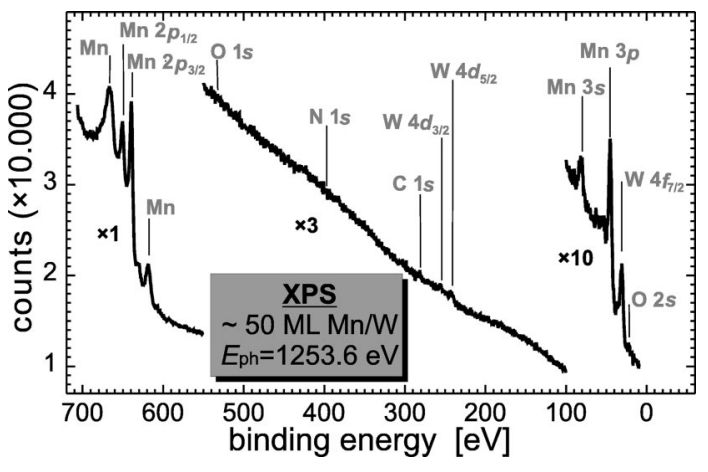

FIG. 1. XPS spectrum of 50土5 ML Mn deposited on a polycrystalline W substrate at room temperature. Due to the roughness of the film some W peaks appear in the spectrum. No significant signal from potential contaminants like $\mathrm{C}$, $\mathrm{O}$, or $\mathrm{N}$ could be detected.

tamination could be found by means of ultraviolet photoemission spectroscopy, Auger-electron spectroscopy, and $\mathrm{X}$-ray photoemission spectroscopy (XPS).

$\mathrm{Mn}$ was evaporated from a tungsten crucible heated by electron bombardment. The evaporant was degassed by prolonged heating up to approximately $1500 \mathrm{~K}$. Higher degassing temperatures could not be realized due to a rapidly growing vapor pressure (above $10^{-2}$ torr), which may result in a destructive glow discharge within the evaporator. During $\mathrm{Mn}$ deposition the pressure remained below $p=2$ $\times 10^{-10}$ torr. Figure 1 shows the XPS spectrum as measured on a $50 \mathrm{ML} \mathrm{Mn}$ film deposited at room temperature on a polycrystalline $\mathrm{W}$ substrate. No hints of any contaminant like, e.g., C, N, or O, are detected.

In fact, two different microscopes and two different $\mathrm{W}(110)$ substrates were used for the experiments reported here. Structural and non-spin-polarized electronic properties of thin Mn films were investigated using a home-built UHVcompatible STM especially designed for thin-film growth studies. ${ }^{45}$ In short, its piezo-driven high-precision coarse approach, which is tilted by $60^{\circ}$ with respect to the tip axis, allows normal film deposition onto the sample when it is retracted from the tip by about $20 \mathrm{~mm}$. This prevents both potential artifacts caused by striking incidence of the metal vapor as well as unwanted tip changes. The coarse motor position accuracy allows one to regain the same particular spot of a sample within a series of evaporation steps. Every cycle of the series requires 30-40 min. For non-spinpolarized measurements we used Pt/Ir tips. This STM is equipped with a sample holder which is large enough to allow the use of a disk shaped tungsten single crystal with a diameter of $10 \mathrm{~mm}$ and a thickness of $0.8 \mathrm{~mm}$. Its surface is electromechanically polished with an average miscut of less than $2^{\circ}$ toward the (110) plane.

Low-temperature measurements were performed instead in a home-built STM which requires a smaller sample size, ${ }^{43}$ i.e., a rectangular $\mathrm{W}(110)$ substrate with the outer dimensions $3 \mathrm{~mm} \times 7 \mathrm{~mm}$. The average miscut of the latter substrate amounts to $0.5^{\circ}$. This microscope was designed for high-spatial-resolution studies of surface magnetism and includes a tip exchange mechanism. ${ }^{43}$ For magnetic measure- ments we used etched W tips which were flashed in vacuo to remove oxide layers. In the metal vapor deposition chamber the tips were magnetically coated with Fe or Gd while held at $300 \mathrm{~K}$, subsequently annealed at $T \approx 550 \mathrm{~K}$ for $4 \mathrm{~min}$, and then transfered into the cryogenic STM. During the measurements, the tip and sample were at a temperature $T=16 \mathrm{~K}$.

All STM images shown below were measured in the constant-current mode of operation. The vertical and lateral sensitivity of the tube scanner was calibrated on monatomic steps of the $\mathrm{W}(110)$ substrate and on the $(15 \times 3)$ unit cell of a carbon-induced reconstruction of the W(110) surface, respectively. ${ }^{44}$ The STM data were plane fitted on atomically flat terraces to correct the tilt of the sample. We enhanced the contrast of the STM images by mixing the tip height $z$ and its derivative with respect to the fast scan direction $x$, i.e., $d z / d x$, at a ratio of $0.1 \quad z: 0.9(d z / d x)$. This image processing suggests to the spectator a topography that is illuminated by an invisible light source from the left. Spectroscopic information is gained by measuring the derivative of the tunneling current $I$ with respect to the applied sample bias $U$. This so-called $d I / d U$ signal is a measure of the local density of states of the sample below the tip apex. ${ }^{46}$ The $d I / d U$ signal is measured by a lock-in technique. After switching off the feedback loop an ac component $\left(U_{\text {mod }} \leqslant 30 \mathrm{mV}\right.$, $\nu \approx 2 \mathrm{kHz}$ ) is added to the gap voltage $U$, which is ramped linearly, and 80-150 values of the lock-in signal are acquired. At the end of the ramp the modulation is switched off and the feedback is reactivated.

\section{RESULTS AND DISCUSSION}

\section{A. Structural properties}

Mn exhibits a great variety of different structural phases. The equilibrium room temperature structure is cubic $\alpha$-Mn which exhibits an untypically large bulk unit cell containing 58 atoms. $^{47}$ In bulk Mn simple cubic phases, namely, facecentered cubic (fcc) $\gamma$-Mn and body-centered cubic (bcc) $\delta$-Mn, are stable only at high temperatures close to the melting point. Another possibility to stabilize $\mathrm{Mn}$ - and any other element-in a nonequilibrium crystalline symmetry is by heteroepitaxial growth on an appropriate substrate. The dense-packed (110) surface of $\mathrm{W}$ is a very popular substrate. $\mathrm{W}$ exhibits a bcc crystal structure and an equilibrium lattice constant $a_{\mathrm{W}}=3.16 \AA$, i.e., the misfit $f$ to $\delta$ - $\mathrm{Mn}\left[a_{\mathrm{Mn}}=2.95\right.$ $\pm 0.03 \AA$ ) (Refs. 48 and 49) $]$ amounts to $f=\left(a_{\mathrm{W}}\right.$ $\left.-a_{\mathrm{Mn}}\right) / a_{\mathrm{Mn}}=(7.3 \pm 1.1) \%$.

The room-temperature growth of the Mn monolayer on a $\mathrm{W}(110)$ substrate is illustrated by the series of six STM images shown in Fig. 2. Figure 2(a) shows the bare W(110) substrate. Ten atomically flat terraces separated by steps of monatomic height can be recognized. Due to a certain fluctuation of the step density a variety of different terrace widths was found. An approximately $100 \mathrm{~nm}$ wide terrace can be seen in the middle of the image. In contrast, the width of terraces is strongly reduced in the upper right and lower left of the image down to about $20 \mathrm{~nm}$. This surface was incrementally exposed for $30 \mathrm{~s}$ to the flux of the Mn evaporator. As can be seen in Fig. 2(b) the first evaporation cycle leads to the nucleation of small islands with a typical length 

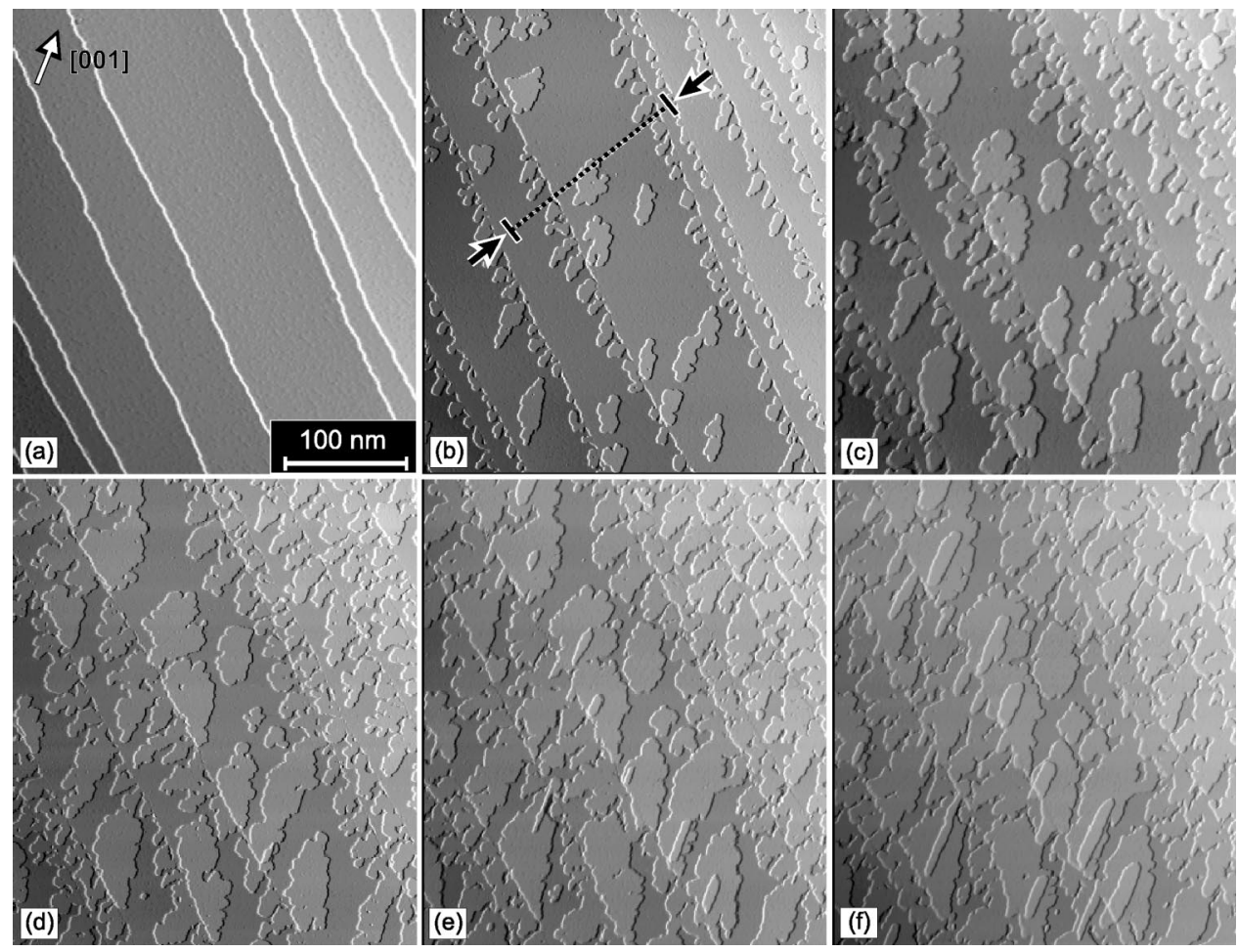

FIG. 2. Topography of the same spot of the sample surface showing (a) the clean W(110) substrate and after the deposition of (b) $0.17 \mathrm{ML}$, (c) $0.34 \mathrm{ML}$, (d) $0.51 \mathrm{ML}$, (e) $0.68 \mathrm{ML}$, and (f) 0.85 ML. The measurement parameters were $U=+0.2 \mathrm{~V}$ and $I$ $=0.2 \mathrm{nA}$. of a few tens of a nanometer and which are elongated along the [001] direction of the substrate. In order to allow a quantitative analysis of the apparent height of the Mn monolayer on $\mathrm{W}(110)$ in STM images we have drawn a line section across one particular island in Fig. 2(b) indicated by a hatched line. The result is plotted in Fig. 3. Based on a comparison with the monatomic step height of the W(110) surface, which amounts to $2.24 \AA$, the apparent height of the Mn monolayer can be determined to $2.36 \pm 0.02 \AA .{ }^{50}$ The step edges are decorated by smaller Mn fingers. The apparent coverage amounts to $0.17 \pm 0.01 \mathrm{ML}$. Since the nucleation density is very low $\left(\approx 1\right.$ island per $\left.4000 \mathrm{~nm}^{2}\right)$ almost no island can be found on terraces that are narrower than about $25 \mathrm{~nm}$ [cf. Fig. 2(b)]. Significant nucleation of second monolayer islands on top of islands of the first monolayer can be observed at a coverage of $0.68 \mathrm{ML}$ in Fig. 2(e). Obviously, the tendency to grow anisotropically along the [001] direc-

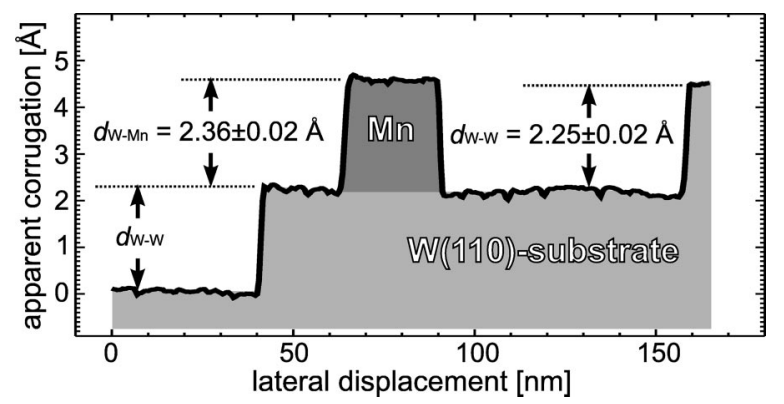

FIG. 3. Single line section (black line) drawn along the hatched line in Fig. 2(b). The tungsten substrate and the Mn monolayer have been differently gray shaded. At this particular tunneling parameters ( $U=+0.2 \mathrm{~V}$ and $I=0.2 \mathrm{nA}$ ) the apparent height of the Mn monolayer amounts to $2.36 \pm 0.02 \AA$. tion is much stronger for the second layer than for the first monolayer. It is well known that any relaxation between the adlayer and the substrate as a consequence of different crystallographic properties, i.e., the symmetry or the periodicity of the crystal lattice, leads to the appearance of dislocation lines or networks, as, e.g., found for Fe films on W(110) either by LEED (Ref. 51) or in STM studies. ${ }^{52,53}$ Instead, in the case of the Mn monolayer on W(110) we found no hint of dislocations either in the STM topographs [Fig. 2(b)-2(f)] or in the LEED pattern, which is free of superstructures and satellite spots. This can be recognized in Fig. 4, showing three LEED patterns which were observed at different Mn

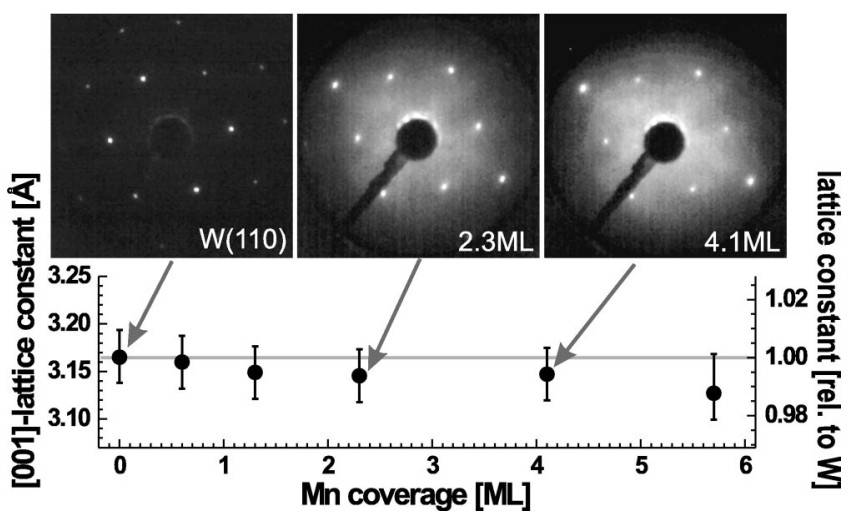

FIG. 4. Plot of the lattice constant $a_{\text {cub }}$ along the [001] direction versus the amount of Mn deposited on a W(110) substrate as determined from the LEED pattern at $E_{p}=160 \mathrm{eV}$. $a_{\text {cub }}$ remains constant within the error bar. The inset shows LEED patterns at three selected coverages. No satellite spots were found at any coverage. Instead, with increasing $\mathrm{Mn}$ coverage the diffuse background becomes more and more intensive, indicating pseudomorphic growth at low coverage and poor medium-range order at higher coverage. 
coverages. We have also analyzed the spot spacing of the LEED pattern for Mn coverages of up to 5.7 ML quantitatively (Fig. 4). All data were calibrated to the bare W substrate, which is known to exhibit a cubic lattice constant $a_{\text {cub }}=3.165 \AA$. Although the data points suggest a certain tendency toward relaxation the determined values for $a_{\text {cub }}$ are constant within the error bar. In addition to a slight broadening of the LEED spots along the [1 $1 \overline{1} 0]$ direction, which is caused by the reduced width of the Mn islands along this direction, the LEED pattern is the same as for the bare substrate even at a coverage of 4.1 ML.

Based on the LEED and STM data presented here we can conclude that the first and second Mn layers grow pseudomorphically on $\mathrm{W}(110),{ }^{54}$ i.e., $\mathrm{Mn}$ mimics the bcc symmetry as well as the lattice constant of the underlying substrate in spite of the enormous tensile strain.

\section{B. Magnetic ground state and interlayer distance}

\section{Theory}

As already mentioned above, the equilibrium roomtemperature structure of $\mathrm{Mn}$ is cubic $\alpha$-Mn. $\alpha$-Mn exhibits a complex noncollinear magnetic structure below its critical bulk temperature $T_{c}^{b}=100 \mathrm{~K} \cdot{ }^{55,56}$ We have shown above that $\delta$-Mn can be stabilized by pseudomorphic growth on W(110) (cf. Figs. 2-4). The magnetic structure of $\delta$-Mn in reduced dimensions is unknown a priori. In order to find the magnetic ground state we have considered three possible spin structures of a Mn monolayer on W(110), which are schematically represented in Fig. 5: (a) ferromagnetic, (b) $c$ (2 $\times 2$ ) antiferromagnetic with an antiferromagnetic coupling between all nearest-neighbor atoms, and additionally (c) $p(2 \times 1)$ antiferromagnetic, where two nearest-neighbor atoms couple ferromagnetically while the other two couple antiferromagnetically. For all configurations the equilibrium interlayer distance between $\mathrm{Mn}$ and $\mathrm{W}$ was determined by total energy minimization [Fig. 6(a)]. By comparing the total energies of the three magnetic structures we conclude that the $c(2 \times 2)$ AFM configuration [Fig. 5(b)] is the magnetic ground state structure, i.e., at low temperatures a checkerboard arrangement of magnetic moments occurs. The energy of the $c(2 \times 2)$ AFM configuration is $188 \mathrm{meV}$ and $102 \mathrm{meV}$ per Mn atom lower than the ferromagnetic [Fig. 5(a)] or the $p(2 \times 1)$ AFM [Fig. 5(c)] state, respectively.

These energy differences between the different magnetic configurations are larger than those published in Ref. 21, which were based on the LSDA. Applying the LSDA, the Mn-W interlayer distances are $1.89 \AA, 1.93 \AA$, and $1.99 \AA$ for the FM, the $p(2 \times 1) \mathrm{AFM}$, and the $c(2 \times 2) \mathrm{AFM}$ state, respectively, ${ }^{57}$ i.e., considerably smaller than the GGA results of $2.04 \AA$, $2.12 \AA$, and $2.14 \AA$. Due to the strong $3 d-5 d$ hybridization between $\mathrm{Mn}$ and $\mathrm{W}$ atoms, this change in the interlayer distance of about $0.15 \AA$ changes the magnetic moment of Mn by about $0.45 \mu_{B}$ and thus the energy differences between the magnetic states. As shown in Fig. 6(b) using the GGA, the equilibrium Mn magnetic spin moments are $2.97 \mu_{B}, \pm 3.20 \mu_{B}$, and $\pm 3.32 \mu_{B}$ for the FM, the $p(2 \times 1) \mathrm{AFM}$, and the $c(2 \times 2) \mathrm{AFM}$ configurations, re-

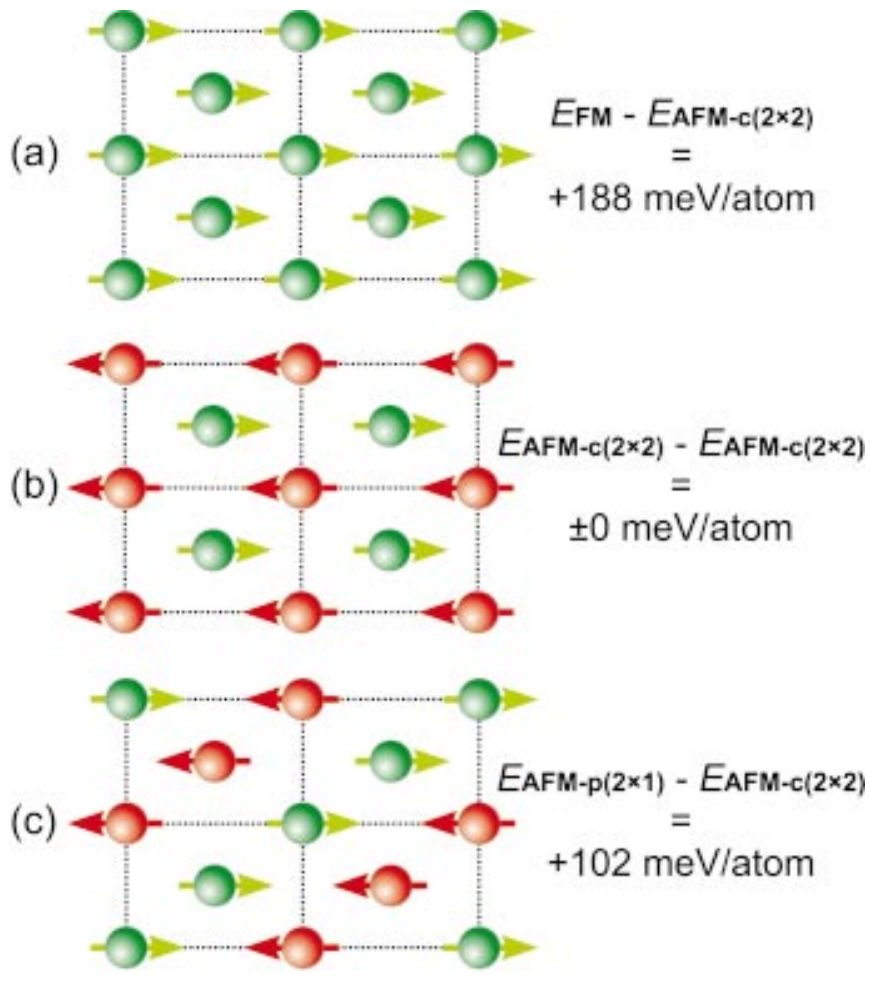

FIG. 5. (Color) Three different possible magnetic configurations of the Mn monolayer on $\mathrm{W}(110)$ that have been considered in this publication: (a) ferromagnetic, (b) $c(2 \times 2)$ antiferromagnetic, and (c) $p(2 \times 1)$ antiferromagnetic.

spectively. Also the $\mathrm{W}$ atom at the interface exhibits a small induced magnetic moment of about $0.2 \mu_{B}$ and $\pm 0.25 \mu_{B}$ for the FM and the $c(2 \times 2)$ AFM states, respectively. To examine the role of the GGA on the magnetic moments we have calculated the magnetic moment of $\mathrm{Mn}$ and $\mathrm{W}$ in the $c(2$ $\times 2$ ) AFM state with the LSDA but for the interlayer distance as calculated with the GGA functional. We found a magnetic moment of $3.18 \mu_{B}$ for $\mathrm{Mn}$ and $\pm 0.2 \mu_{B}$ for the $\mathrm{W}$ interface atom. Thus the main change of the magnetic moments comes from the different interlayer distance due to the improved GGA.

We determined also the easy axis of the magnetization $\hat{M}$ which minimizes the magnetic anisotropy energy $E_{\mathrm{MAE}}(\hat{M})$. Due to the symmetry of the (110) unit cell the easy axis assumes one of three possible magnetization directions, the out-of-plane direction [110] along the surface normal, or the in-plane directions along the long [1 10$]$ or short [001] axis of the surface unit cell. The magnetic anisotropy energy consists of two contributions, the magnetocrystalline anisotropy and the dipolar interaction. For antiferromagnets, the latter is very small and is neglected. The former requires knowledge of the electronic structure including the spin-orbit interaction and it is calculated as the difference of total energies determined for different magnetization directions $\hat{M}$. From these calculations we conclude that for the ground state the magnetic moments of the $c(2 \times 2)$-antiferromagnetic structure have an in-plane orientation along the [1 10$]$ direction (long side of the surface unit cell). This is the easy axis. The en- 

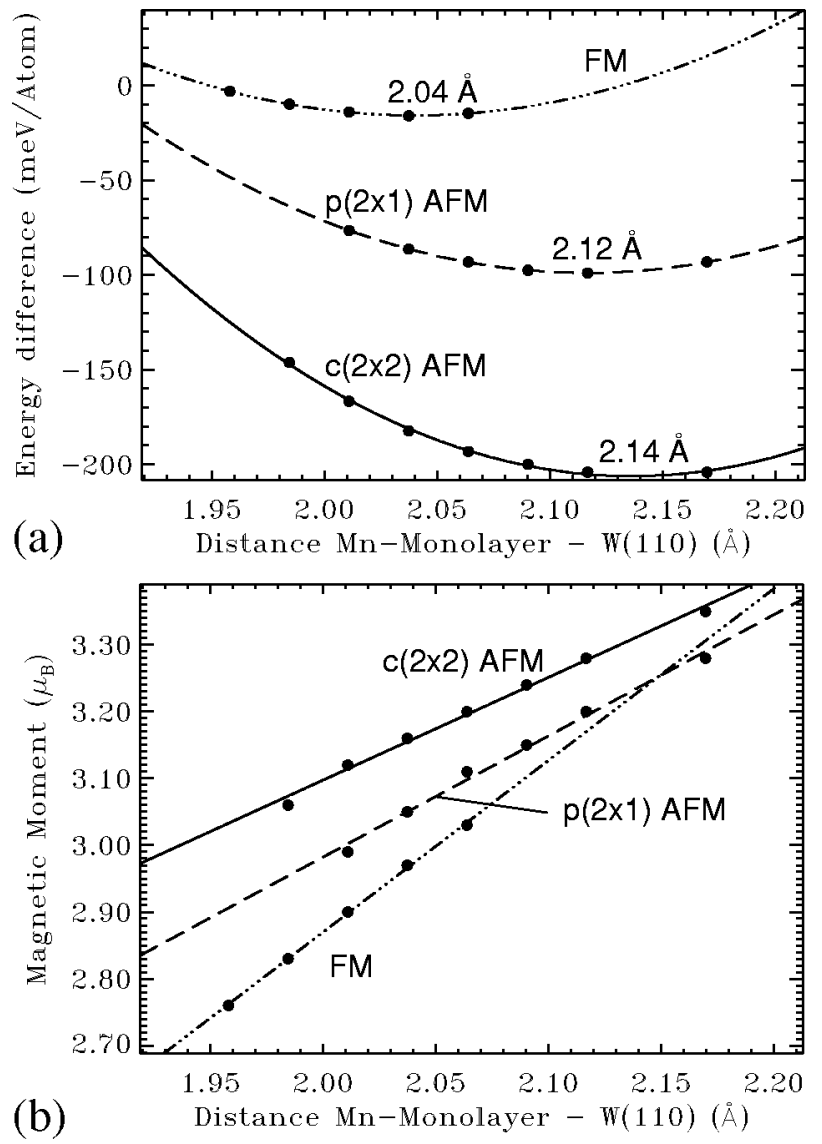

FIG. 6. The interlayer distance (a) and magnetic moments (b) of the Mn monolayer on $\mathrm{W}(110)$ as determined by total energy minimization for the three different magnetic configurations shown in Fig. 5.

ergy difference between the easy axis and the hard axis, which is the out-of-plane orientation of the magnetic moments, amounts to about $1.3 \mathrm{meV}$ per atom calculated employing the LSDA functional and $1.55 \mathrm{meV}$ calculated with the GGA functional, both for the equilibrium GGA interlayer distance. Calculated in the LSDA (GGA) with respect to the easy axis the anisotropy energy along the [001] axis is about 0.44 (0.80) meV higher.

\section{Experiment}

Apparently, the calculated interlayer distance of about $2.14 \AA$ for the $c(2 \times 2)$ AFM configuration [Fig. 6(a)] does not agree with the apparent step height of $2.36 \pm 0.02 \AA$ as measured by STM (cf. Fig. 3). As we will point out in the following this discrepancy of about $0.22 \AA$ between theory and experiment can at least in part be explained by different work functions $\Phi$ of the W(110) substrate and the Mn monolayer. The work function of a surface is related with the barrier height in a tunneling experiment. It can be determined by a measurement of the tunneling current $I$ as a function of the tip-sample distance $z$. This experiment was performed by stabilizing the tip at a sample bias voltage $U_{\text {stab }}$ $=3 \mathrm{~V}$ and a tunneling current $I=10 \mathrm{nA}$ above the sample surface. Then the feedback loop is switched off and the tip is

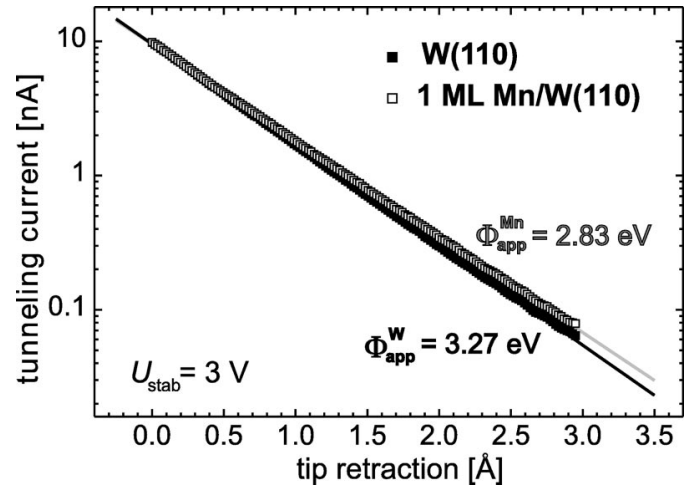

FIG. 7. Distance-dependent STM current as measured above the bare W(110) substrate and the Mn monolayer on W(110).

retracted from the sample surface by $3 \AA$. During the tip retraction the decay of the tunneling current is measured. Figure 7 shows a semilogarithmic plot of $I$ versus the tip retraction (relative to the stabilization point). Obviously, the graph fits the expected exponential behavior

$$
I \propto \exp (-2 \kappa z)
$$

with

$$
\kappa=\frac{\sqrt{2 m \Phi_{\text {app }}}}{\hbar},
$$

where $\Phi_{\text {app }}$ is the apparent barrier height. We can extract the effective local barrier height by

$$
\Phi_{\mathrm{app}}=\frac{\hbar^{2}}{8 m}\left(\frac{d \ln I}{d z}\right)^{2} .
$$

Analysis of the data of Fig. 7 gives $\Phi_{\mathrm{app}}^{\mathrm{W}}=3.27 \mathrm{eV}$ and $\Phi_{\mathrm{app}}^{\mathrm{Mn}}=2.83 \mathrm{eV}$. The bias and energy dependence of the barrier height can be corrected ${ }^{46}$ by

$$
\Phi=\Phi_{\mathrm{app}}+\frac{e U}{2},
$$

resulting in $\Phi_{\text {expt }}^{\mathrm{W}}=4.77 \mathrm{eV}$ and $\Phi_{\text {expt }}^{\mathrm{Mn}}=4.33 \mathrm{eV}$. Although the experimental result is somewhat lower than the calculated work functions of the W(110) surface, $\Phi_{\text {theo }}^{\mathrm{W}}$ $=5.06 \mathrm{eV}$, and of the $c(2 \times 2)$ AFM Mn monolayer on $\mathrm{W}(110), \Phi_{\text {theo }}^{\mathrm{Mn}}=4.56 \mathrm{eV}$, the tendency, i.e., a reduction of the work function above the Mn monolayer with respect to the bare W(110) substrate, is consistent. As a consequence the tip-sample distance at a constant current will depend on whether the tip is positioned above the clean W(110) surface or above the Mn monolayer, i.e., $z_{\mathrm{W}}$ and $z_{\mathrm{Mn}}$ will be different. More precisely, for the constant-current mode of operation $\left(I=\right.$ const) insertion of the values $\Phi^{\mathrm{W}}, \mathrm{Mn}_{\text {expt }}$ in Eq. (8) gives the relation

$$
\frac{z_{\mathrm{Mn}}}{z_{\mathrm{W}}}=\frac{\sqrt{\Phi_{\mathrm{expt}}^{\mathrm{W}}}}{\sqrt{\Phi_{\mathrm{expt}}^{\mathrm{Mn}}}}=1.05 .
$$


In other words, the tip-sample distance is about 5\% larger with the STM tip above the Mn monolayer on W(110) than above the clean W(110) substrate. However, the absolute tipsample distance in our experiment is unknown. As an estimate we may use the result of Ref. 58 which was obtained by cross-sectional imaging of a vacuum tunnel junction between two gold electrodes by means of high-resolution transmission electron microscopy. The authors of Ref. 58 revealed that at the tunneling parameters $U=+0.1 \mathrm{~V}$ and $I$ $=1.4 \mathrm{nA}$ the distance between the electrodes amounts to $10 \pm 1 \AA$. In our experiment the same tip-sample distance would lead to an apparent $\mathrm{Mn}$ monolayer step height which-compared to the real interlayer distance-is exaggerated by about $0.5 \AA$. This value is much larger than the observed difference of $0.22 \AA$ between experiment and calculation. Up to now we have neglected, however, that there are also electronic effects which contribute to the tunneling current as a prefactor in Eq. (6). As will be discussed in the next section the clean $\mathrm{W}(110)$ surface possesses a larger electronic prefactor than the Mn monolayer. Thus the work function effect is partly compensated and a reduction of the discrepancy between calculated and measured interlayer distance is expected. In conclusion, the difference of $0.22 \AA$ is in reasonable agreement with our expectation.

\section{Electronic properties}

It is clear that the electronic structure depends on the magnetic ground state. In turn, the knowledge of the electronic structure, e.g., measured by STS, combined with a thorough comparison with the electronic structure calculation may lead to the determination of the magnetic ground state. In the following we will perform such an analysis.

The top part of Fig. 8 shows the calculated band structure of the $c(2 \times 2) \mathrm{AFM}$ ground state of a Mn monolayer on $\mathrm{W}(110)$ plotted along the $\bar{\Gamma}-\overline{\mathrm{M}}$ direction of the surface Brillouin zone. In the lower part of Fig. 8 the spin-resolved local density of states is displayed within the muffin-tin spheres of the Mn atoms. Since all Mn atoms are chemically equivalent but couple antiferromagnetically, i.e., the quantization axis flips from atom to atom, the majority LDOS of a selected atom is identical to the minority LDOS of the neighboring one, and vice versa. Therefore, the sum of the majority and minority LDOS's is equal for all atoms. For further discussion we introduce a global quantization axis for the unit cell and the entire system, respectively, which we associate arbitrarily with one type of atom, say the atom with the magnetization axis pointing in the $[1 \overline{1} 0]$ direction.

However, in a STM experiment it is not the LDOS within the topmost layer that is measured but the LDOS some 5 to $10 \AA$ Above it [see Eq. (1)]. In order to discuss the expected results of a STM experiment from the calculated data we have included in Fig. 8 plots of the first three star coefficients $n_{0}(z, \epsilon), n_{1}(z, \epsilon)$, and $n_{2}(z, \epsilon)$ at a distance of $z=0.0 \AA$ for both spin directions. Note that we have plotted only the spinsummed value in the case of $n_{0}$ as the majority and minority contributions summed up over the two atoms of the unit cell are the same. The exponential decay of the star functions with increasing order is easily seen from the $y$ scale of Fig. 8 .

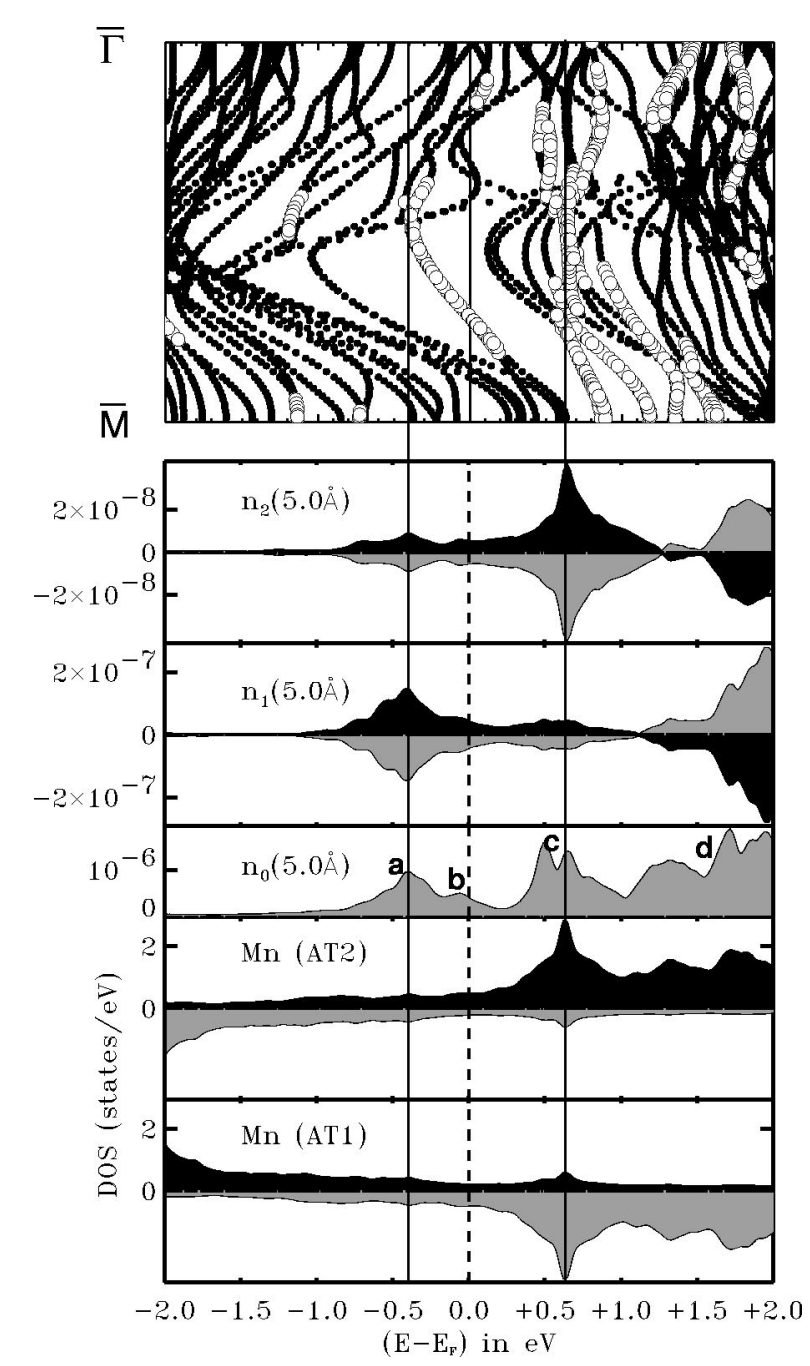

FIG. 8. Comparison of the band structure, zeroth, first, and second star coefficients, and the LDOS of the two chemically equivalent Mn atoms (AT1, AT2) of the $c(2 \times 2)$ AFM Mn monolayer on $\mathrm{W}(110)$. In the band structure, states that are localized by more than $60 \%$ in the Mn monolayer and the vacuum region are marked by open circles. The band structure is shown for one spin contribution only since the other is identical. The first and second star coefficients and the LDOS are shown for the majority (minority) contributions denoted by dark (gray) shaded areas with respect to the spin-quantization axis of atom one (AT1), which we assume here to be the global magnetization axis. For atom 2 (AT2), minority electrons related to the global spin-quantization axis are the majority electrons in the local quantization axis.

In the following we focus on a discussion of scanning tunneling spectroscopy measurements where the differential conductivity $d I / d U$ is the experimentally accessed quantity. In the model of STM described in Sec. II B the differential conductivity is given by

$$
\frac{d I}{d U}(U) \propto n\left(\mathbf{r}_{\|}, z, \epsilon_{F}+e U\right)
$$

for a non-spin-polarized measurement. Normally, the resolution of such a measurement is above the atomic scale and therefore the LDOS can be replaced by its laterally constant 


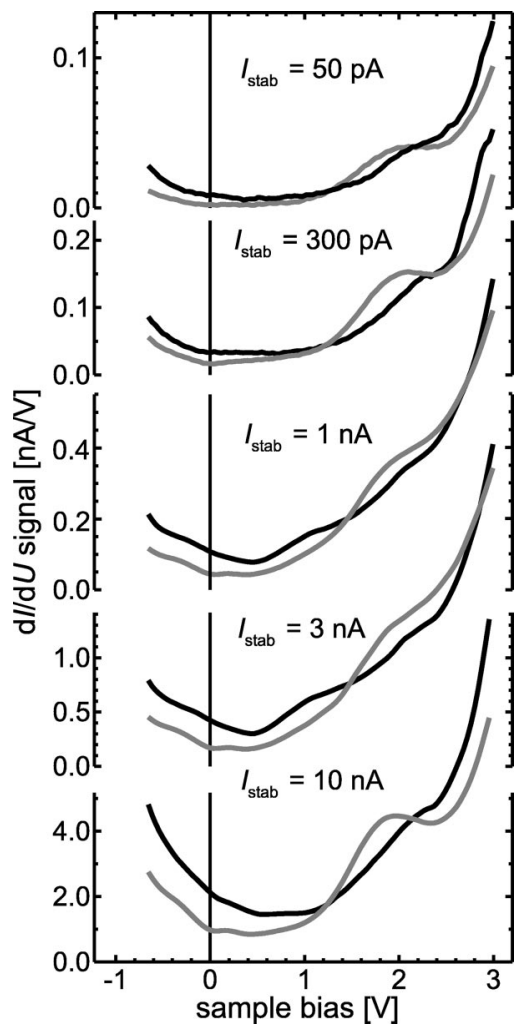

FIG. 9. Tunneling $d I / d U$ spectra of the bare W(110) substrate (black) and a Mn monolayer (gray) as measured with different stabilization currents $I_{\text {stab }}\left(U_{\text {stab }}=+3 \mathrm{~V}\right)$. A peak at $U=+1.8 \mathrm{~V}$ is observed above Mn. Regarding the peak position the spectra are independent of $I_{\text {stab }}$.

part, which is the zeroth star coefficient. Thus we can directly compare the zeroth star coefficient with measured $d I / d U$ spectra. Based upon this model the calculation presents four features marked by small letters in Fig. 8. There are peaks at $a-0.4 \mathrm{eV}, b-0.06 \mathrm{eV}$, and at $d+1.7 \mathrm{eV}$, as well as a double peak structure $c$ with peaks at $+0.5 \mathrm{eV}$ and $+0.65 \mathrm{eV}$.

The theoretical results can be checked by scanning tunneling spectroscopy. We have performed measurements of the differential tunneling conductivity $d I / d U$ on both the bare W(110) substrate as well as on Mn monolayer sites by using a sample surface with a topography similar to Fig. 2(c). The results are shown in Fig. 9. In order to minimize any interaction between the tip and the sample we have chosen a very small stabilization current $I_{\text {stab }}=50 \mathrm{pA}$ in the first measurement $\left(U_{\text {stab }}=+3 \mathrm{~V}\right)$. While the $\mathrm{W}(110) d I / d U$ spectrum is featureless, the Mn monolayer spectrum exhibits a clear shoulder at $U=+1.8 \mathrm{~V}$. This shoulder may be identified with the peak labeled $d$ in the laterally constant part of the calculated DOS, $n_{0}$ in Fig. 8, which has been predicted to occur at an energy of $1.7 \mathrm{eV}$ above the Fermi level. One crucial question that is often asked is whether tip and sample can be regarded as independent electronic systems even at low tip-sample distance, i.e., at low bias voltages and high tunneling currents, or whether the measured eigenstates reflect the properties of the tip-sample ensemble. According to textbook tunneling theory it is expected for the case of non-

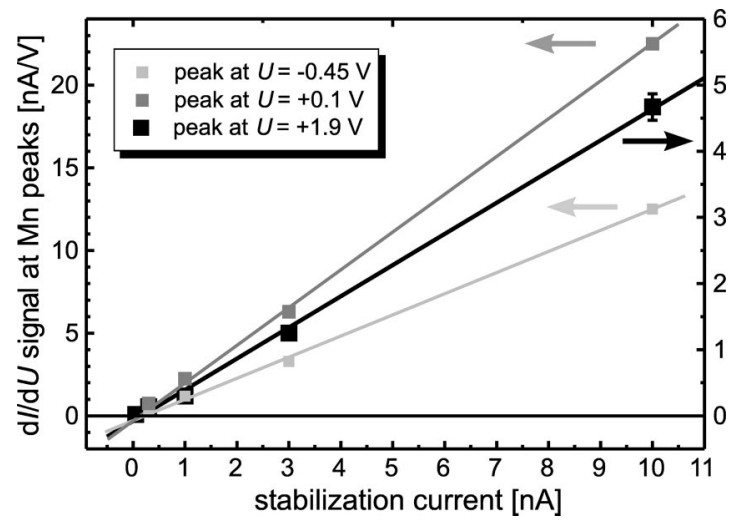

FIG. 10. Height of the $d I / d U$ peak measured above the Mn monolayer on $\mathrm{W}(110)$. In the semilogarithmic representation a linear behavior can be recognized, in agreement with the TersoffHamann model.

interacting tip and sample that the tunneling current $I$ as well as the differential conductivity $d I / d U$ will increase exponentially with decreasing tip-sample distance. In other words, a linear relationship is expected in a plot of the $d I / d U$ signal at the Mn peak versus $I_{\text {stab }}$. Consequently, any distortion of the sample's electronic structure by the close tip or vice versa must result in a nonlinear contribution.

We have answered this question for the sample investigated here by performing a systematic study of the dependence of the $d I / d U$ spectra as function of the stabilization current $I_{\text {stab }}$. Although at higher stabilization currents the measured differential conductivity dramatically increases (cf. the $y$ scale of $d I / d U$ in Fig. 9), the position of the peak remains almost unchanged up to $I_{\text {stab }}=10 \mathrm{nA}$. As can be seen in Fig. 10 a linear relationship is perfectly fulfilled within the measurement accuracy, indicating that tip and sample can indeed be regarded as independent electronic systems.

So far only one of the four features that were indicated in the DOS of the Mn monolayer could be confirmed experimentally. We have to keep in mind, however, that due to the high bias voltage $(U=+3 \mathrm{~V})$ at the starting point of the voltage ramp the features in the DOS which are close to the Fermi level are hardly visible. In order to enhance the sensitivity in this energy range we have performed further STS measurements with $U=+0.9 \mathrm{~V}$ as the starting point of the voltage ramp. A representative result is shown in Fig. 11. Now, two additional peaks at about $U=-0.45 \mathrm{~V}$ and $U$ $=+0.1 \mathrm{~V}$ are visible. They can be identified with peak $a$ and $b$ in Fig. 8. Similar to the measurements described above (Fig. 9) these STS experiments have also been performed with different stabilization currents (not shown here). Again, Fig. 10 reveals a linear relation between the $d I / d U$ signal and $I_{\text {stab }}$. While the energetic position of peak $a$ is in almost perfect agreement with the theoretical prediction, there is a small discrepancy between the position of peak $b$ at $0.1 \mathrm{eV}$ in the $d I / d U$ spectrum and the theoretical result located at $-0.06 \mathrm{eV}$. We could not find any peak in the experimental data that could be correlated to the double peak structure $c$ in Fig. 8 .

Obviously, we find a good overall agreement between the 


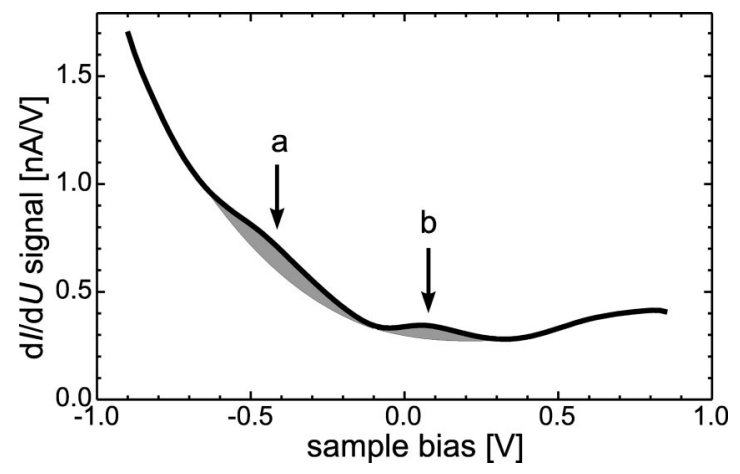

FIG. 11. $d I / d U$ spectrum measured above the Mn monolayer on $\mathrm{W}(110)$. The stabilization current was $I=0.3 \mathrm{nA}$.

calculated local density of states peaks and the measured ST spectra for most of the calculated peaks. However, there are limits in calculating ST spectra on the basis of the model of Tersoff and Hamann ${ }^{32}$ due to some simplifying assumptions. The most problematic assumption is that of a constant tip DOS over large energy ranges, which clearly breaks down at large bias voltages. Further, one cannot assume a priori that the single particle energies calculated within density functional theory describe excitations at energies far from the Fermi energy with a good accuracy. We believe that the breakdown of these approximations is the reason for the appearance of a double peak structure in the calculation, which is absent in the experiment.

Although some aspects of the calculated electronic structure of the $c(2 \times 2)$ AFM structure as shown in Fig. 8 are in nice agreement with the measured spectra (Figs. 9 and 11), the agreement is not sufficiently unambiguous to deduce the magnetic structure from such a comparison. In the next section we will show that in spite of these limitations there is an elegant and surprisingly easy way to resolve the magnetic ground state by performing constant-current measurements at low bias voltages using magnetic probe tips.

\section{Magnetic imaging by SP-STM}

So far we have determined the $2 \mathrm{D}$ antiferromagnetic ground state of a Mn monolayer on W(110) on the basis of ab initio calculations (Sec. III B) and compared its calculated electronic structure with non-spin-polarized STS measurements (Sec. III C). As we pointed out at the end of the previous section it is difficult to determine the magnetic ground state from comparing non-spin-polarized ST spectra with electronic structure calculations within the Tersoff-Hamann model. However, we have already shown in previous publications ${ }^{21,29}$ how one can use the constant-current mode of a SP-STM to image magnetism at the atomic scale. The principle of SP-STM on the atomic scale is based on the exponential decay of star coefficients with increasing length of the reciprocal lattice vector. ${ }^{21,29}$ It is of general validity and it may lead to the understanding of many more magnetic structures on this shortest possible length scale in the future. In the following we illustrate the principle for a Mn monolayer on $\mathrm{W}(110)$.

In the case of a vanishing spin polarization of the tip, e.g., in the case of a tungsten tip, the tunneling current is given by (a)

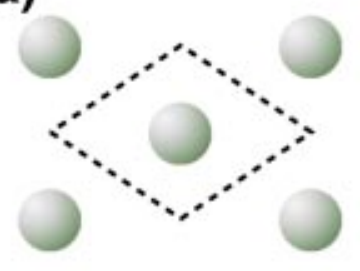

(b)

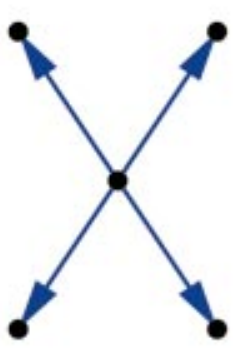

(c)

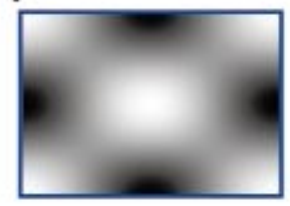

(d)

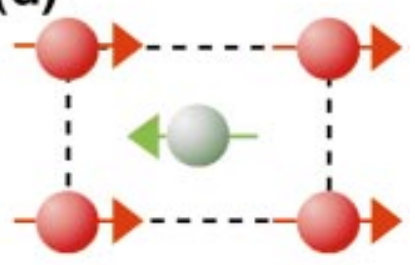

(e)

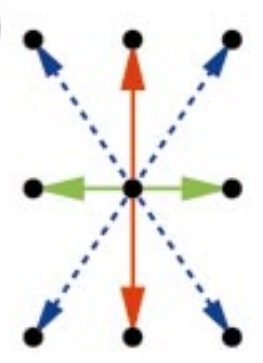

(f)

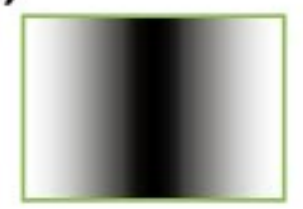

FIG. 12. (Color) Lattice (a),(d), shortest reciprocal lattice vectors (b), (e), and 2D star functions, i.e., the expected STM images (c),(f), associated with the shortest reciprocal lattice vectors of the chemical and magnetic unit cells of a Mn monolayer on W(110), respectively. Note that (e) contains the shortest vectors of the chemical unit cell (dashed) and the two inequivalent pairs of additional vectors due to the magnetic superstructure. In our notation (f) and (c) represent the first and third star functions, respectively.

the spin-summed vacuum LDOS of a Mn monolayer on $\mathrm{W}(110)$ [see Eq. (1)]. This is given by the sum of the vacuum LDOS of the majority and minority electrons as expressed in Eq. (2). From Fig. 8 we find that the first and second star coefficients of the spin-summed LDOS [see Eq. (2)] vanish as the majority and minority contributions possess the same values with opposite sign. This is due to the fact that the first and second star functions distinguish between the two magnetically inequivalent $\mathrm{Mn}$ atoms (see also Fig. 12). As they possess magnetic moments of opposite direction but the same magnitude the first two star coefficients differ only by sign also. For the third star function the two $\mathrm{Mn}$ atoms of the magnetic unit cell are equivalent-it displays the chemical unit cell (see also Fig. 12). Thus the third star coefficient does not vanish for the non-spin-polarized measurement and dominates the STM image. If the tip has a nonvanishing spin polarization, on the other hand, and the angle $\theta$ between the magnetization axes of tip and sample is not equal to $90^{\circ}$, then we also need to take into account the LSDOS [Eq. (3)] of the sample. This is clearly not equal to zero for the first and second star coefficients and thus (because of the exponential dependence with increasing length of $\mathbf{G}_{\|}^{S}$ ) the SPSTM image will be dominated by the first star function.

We can also take a different point of view and start the argument by recalling that according to Eq. (5) the star func- 
tion with the lowest non vanishing reciprocal lattice vector dominates the STM image. If we image Mn on W(110) with a nonmagnetic tip all atoms are equivalent (see the spinsummed DOS of the Mn atoms in Fig. 8), and the chemical unit cell is diamond shaped [Fig. 12(a)]. The four smallest reciprocal lattice vectors of this cell, all related by symmetry operations, are displayed in Fig. 12(b). The corresponding 2D star function [the third star function in the $c(2 \times 2)$ surface unit cell in our discussion above] resulting from the superposition of the four corresponding plane waves represents the expected STM topography [Fig. 12(c)].

However, any magnetic superstructure such as, e.g., the predicted antiferromagnetism of a Mn monolayer on W(110) [cf. Fig. 5(b)], lowers the translational symmetry of the underlying structural (chemical) lattice. In particular, the chemical and the magnetic unit cells of $\mathrm{Mn} / \mathrm{W}(110)$ are symbolized by broken lines in Figs. 12(a) and 12(d), respectively. Then, the additional tunneling current due to spin-polarized electrons $I_{P}$ is sensitive to the unit cell of the superstructure [Fig. 12(d)]. Therefore, smaller reciprocal lattice vectors become accessible [Fig. 12(e)]. Since these possess exponentially larger coefficients [see Eq. (5)] they dominate the STM image even in the case of small effective spin polarization, for example, if the angle $\theta$ is close to $90^{\circ}$. Thus the corrugation amplitude $\Delta z$ (the maximum difference in tip height while it scans the surface) is directly proportional to the spin polarization of the tip and the sample, $\Delta z \sim P_{T} P_{S} \cos \theta$. As a result, a stripe pattern [Fig. 12(f), which represents the first star function] without any chemical background is expected to be seen in the experiment due to the smallest reciprocal superlattice vector.

As we will show in the following this theoretical prediction, which is based on general arguments and confirmed by explicit $a b$ initio calculation, has been experimentally verified by atomic scale STM measurements using probe tips covered with different magnetic materials, i.e., Fe and Gd. The lateral extension of Mn monolayer islands can be enhanced by growing thin films at elevated substrate temperatures $T_{\text {sub }}$. As shown in the STM topograph of Fig. 13 this preparation procedure prevents the nucleation of second monolayer patches. As we zoom onto an atomically flat area using a pure flashed $\mathrm{W}$ tip the atomic structure of the $\mathrm{Mn}$ monolayer on $\mathrm{W}(110)$ becomes visible [Fig. 14(a)]. With a pure (non-spin-polarized) W tip we are not sensitive to the spin of the tunneling electrons. Consequently, we cannot detect the modulation of the spin polarization of the Mn atoms within the antiferromagnetic unit cell. Instead, we measure the total density of states, which is equal above both atoms, and the resulting image [Fig. 14(a)] shows the chemical unit cell of Fig. 12(a). Three single line sections drawn along the [11̄0] direction are plotted in Fig 14(b). The measured corrugation amounts to $20-30 \mathrm{pm}$. If, however, a magnetic probe tip is used the magnetic superstructure dominates the image as can be seen in Fig. 14(c), which was measured with an Fe-coated tip. This result is in qualitative agreement with the theoretical prediction explained above.

In order to allow a more quantitative comparison between theory and experiment we have calculated the corrugation amplitude as a function of the applied bias voltage (Fig. 15).

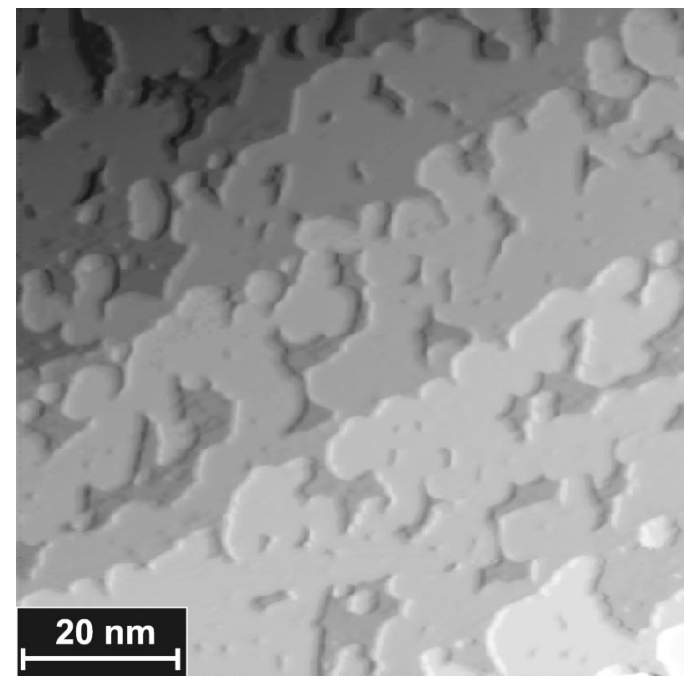

FIG. 13. Topography of a submonolayer Mn film grown on $\mathrm{W}(110)$ with the substrate held at elevated temperatures ( $T$ $=750 \mathrm{~K}$ ). Due to the enhanced mobility of the Mn adatoms, the nucleation of second monolayer islands is avoided.

The contributions from the majority and minority spin states are exactly the same except for the sign of the corrugation amplitude. In the present case the sign denotes that the rows of either ferromagnetic or antiferromagnetic $\mathrm{Mn}$ atoms with respect to the tip magnetization are imaged as protrusions. Thus with a non-spin-polarized STM tip the net corrugation amplitude of the stripe pattern vanishes, and the diamond shaped pattern of the chemical unit cell is observed [cf. Fig. 14(a)]. Although the qualitative agreement of experiment and theory are excellent also in the case of a non-spin-polarized measurement the calculated corrugation amplitude is an order of magnitude too low, due to the fact that the third star coefficient is an order of magnitude lower than the second star coefficient as a result of the increased corresponding reciprocal lattice vector. This is a known deficiency of the Tersoff-Hamann model in explaining STM images of closepacked metal surfaces quantitatively. ${ }^{59}$ However, if the tip possesses a nonvanishing spin polarization $P_{T}$, the corrugation amplitude can be readily computed from the two spin contributions. For Fe-coated W tips a value of $P_{T}=-0.4$ was determined in earlier experiments. ${ }^{61,63}$ The data of Fig. 14(c) have been measured at a small bias voltage value of $U=-3 \mathrm{mV}$, i.e., very close to the Fermi level. At this particular bias voltage, Fig. 15 suggests a corrugation amplitude of about $8 \mathrm{pm}$. This result is in reasonable agreement with the experimentally observed magnetic corrugation of about 4 pm of Fig. 14(d). As the distance dependence of the calculated corrugation amplitude is rather small we attribute the remaining discrepancy to a noncollinear orientation between the magnetization axes of tip and sample, i.e., $\cos \theta \neq \pm 1$. Although the tip magnetization is probably in plane it remains another degree of freedom since in the present experimental setup we cannot control the azimuthal angle of the tip magnetization. ${ }^{62}$ In agreement with Fig. 15 we found only a weak dependence of the observed magnetic corrugation am- 


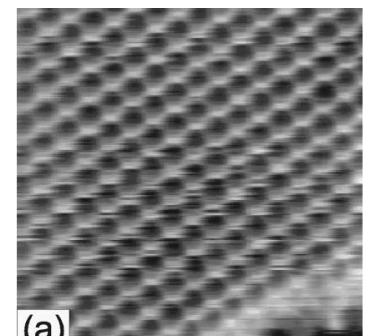

(a)
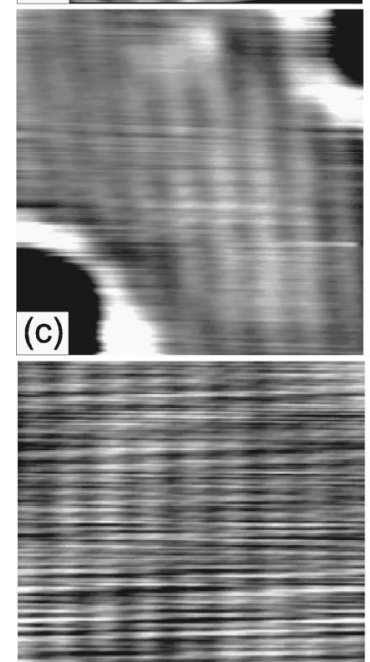

(e)

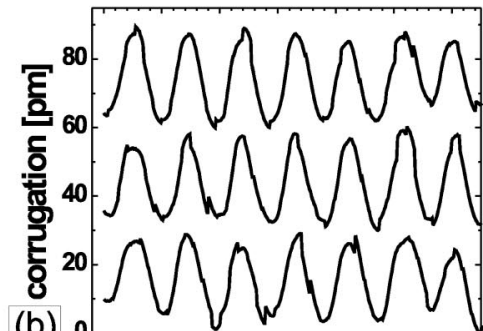

(b) 0
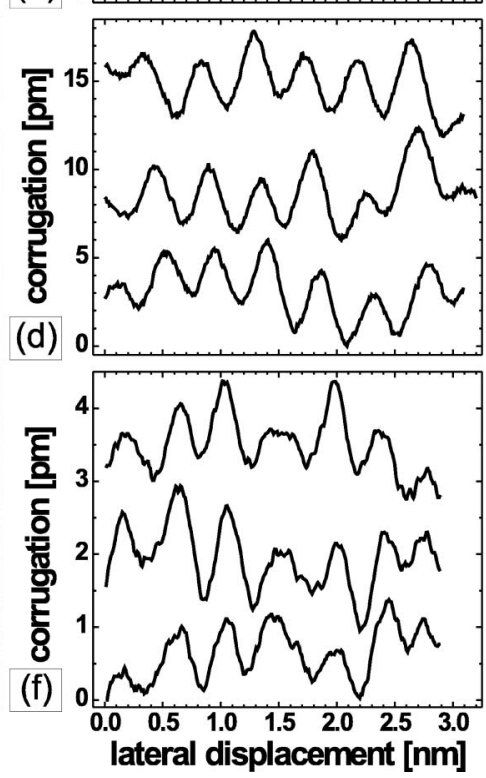

FIG. 14. Atomic scale STM images (raw data) of a Mn monolayer on $\mathrm{W}(110)$ and single line sections drawn along the [1 $\overline{1} 0]$ direction as measured with a bare W- $[(\mathrm{a}),(\mathrm{b})]$, an Fe- $[(\mathrm{c}),(\mathrm{d})]$, and a Gd-coated probe tip [(e),(f)], respectively. At the measurement parameters of $U=-3 \mathrm{mV}$ and $I=40 \mathrm{nA}[(\mathrm{a})-(\mathrm{d})]$ or $U$ $=-10 \mathrm{mV}$ and $I=10 \mathrm{nA}[(\mathrm{e}),(\mathrm{f})]$ the sample could be imaged in many consecutive frame scans without disturbing the sample's surface.

plitude on the applied bias voltage in the range between $-100 \mathrm{meV}$ and $0 \mathrm{meV}$ and no qualitative difference of the images (not shown here).

The strong dependence of the observed magnetic corrugation on the magnetization direction of the tip can be exploited to gain further information on the magnetization direction of the sample. In particular, the use of a tip which exhibits an easy magnetization axis that is almost perpendicular to the sample surface should lead to a much smaller corrugation amplitude than the previous example. As we know from recent measurements, ${ }^{64} \mathrm{~W}$ tips which are coated with $7 \pm 1$ ML Gd are preferrably magnetized along the tip axis, i.e., perpendicular to the sample surface. Indeed, the stripes along the [001] directions which are typical for the SP-STM image of the antiferromagnetic Mn monolayer on $\mathrm{W}(110)$ are only weakly visible [Fig. 14(e)] and the corrugation never exceeded $1 \mathrm{pm}$ [Fig. 14(f)]. Since close to the Fermi level Gd possesses a similar degree of spin polarization as Fe, i.e., $\left|P_{\mathrm{Gd}}\right|=0.4,{ }^{63}$ the results of Fig. 14(c) and 14(e) can be compared directly. The fact that the magnetic stripe pattern is still visible in the SP-STM image-although with an extremely small corrugation-is a result of the non-

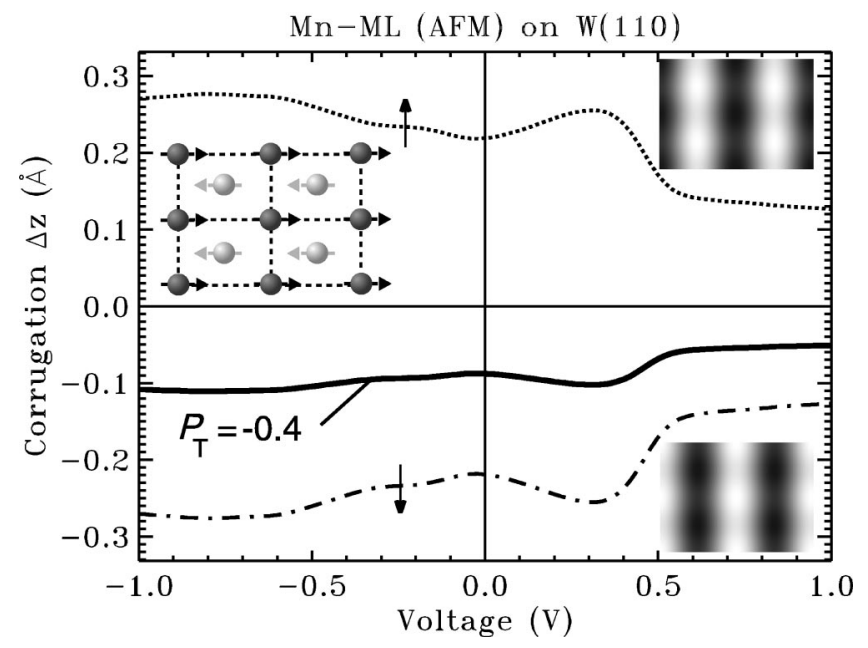

FIG. 15. Calculated corrugation amplitude as a function of the applied bias voltage for the $c(2 \times 2)$ antiferromagnetic configuration of the Mn monolayer on W(110). The dotted and dash-dotted curves denote the two spin contributions while the full line represents the total corrugation amplitude measured with a magnetic tip of spin polarization $P_{T}=-0.4$. A positive (negative) corrugation amplitude is related to imaging the ferromagnetic (antiferromagnetic) Mn atom rows with respect to the tip magnetization as protrusions. The insets show the unit cell of the $c(2 \times 2)$ antiferromagnetic configuration (upper left) and the calculated STM images for $P_{T}>0$ (upper right) and $P_{T}<0$ (lower right).

perfect orthogonal alignment of tip and sample magnetization, probably due to some in-plane component in the magnetization of the tip.

At the beginning of this section we discussed the feasibility of imaging the magnetic superstructure due to the exponential decay of the star coefficients with the vacuum barrier. As the decay of the coefficients to a certain pattern, i.e., the star function $\phi_{s}^{2 \mathrm{D}}\left(\mathbf{r}_{\|}\right)$, of the STM image depends exponentially on the length of the corresponding reciprocal lattice vector $\mathbf{G}_{\|}^{S}$, the smallest nonvanishing vector will dominate the image. However, it must be kept in mind that the electronic structure of a specific sample is contained in the star coefficients. Therefore, it needs to be verified by firstprinciples calculations that there are actually nonzero contributions from electronic states to the pattern with the smallest reciprocal lattice vector. Otherwise, the pattern corresponding to the next larger reciprocal lattice vector will dominate the STM image. In the following we will perform a detailed analysis of the band structure and the star coefficients displayed in Fig. 8. In the course of a thorough analysis the electronic bands that allow the imaging of the magnetic superstructure by means of SP-STM can be identified.

The values of the first and second star coefficients differ for the majority and minority electrons only by their sign since the spin-summed electronic structure of both $\mathrm{Mn}$ atoms is identical. Therefore, in the following we focus on the majority contribution with respect to our chosen quantization axis assuming that the magnetic STM tip used favors this spin channel. Of course, an analogous discussion can be carried over to the minority states with the same result. As can be seen in Fig. 8 the (majority) first star coefficient $n_{\uparrow}^{(1)}(z, \epsilon)$ 

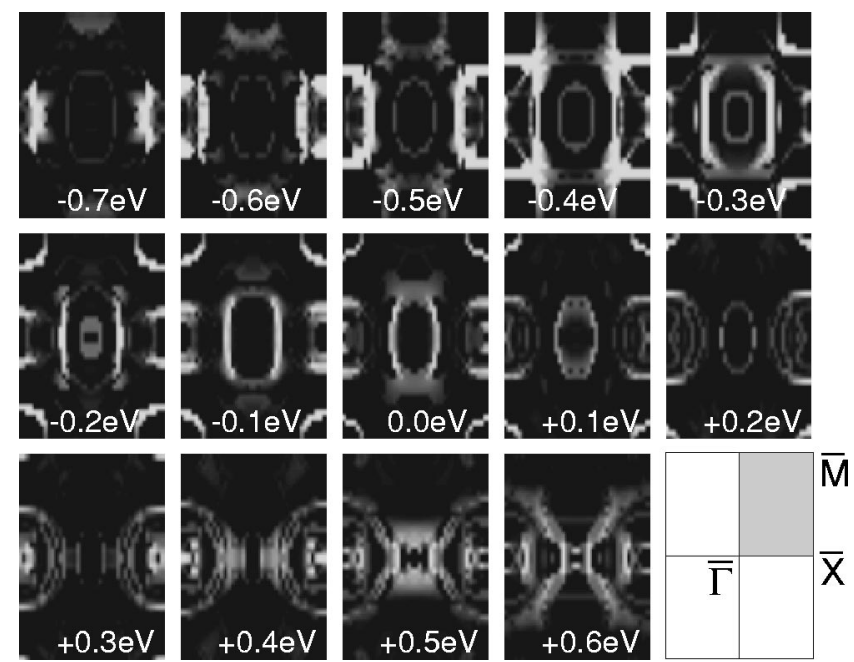

FIG. 16. The majority first star coefficient $n_{\uparrow}^{(1)}(z, \epsilon)$ calculated at a tip-sample distance of $5 \AA$ as a function of the wave vector $\mathbf{k}_{\|}$ in the first Brillouin zone. Calculations were carried out on a mesh with 560 points in the I2BZ (shaded area in the picture of the BZ). Energy intervals of $0.1 \mathrm{eV}$ were chosen. The energies are given relative to the Fermi energy. As the first star coefficient possesses only positive values in the chosen energy range, a gray scale is sufficient. Dark corresponds to zero while the brightest areas give the largest values. The bottom of the surface state band in the $\overline{\Gamma M}$ direction is clearly visible at $\epsilon=\epsilon_{F}-0.4 \mathrm{eV}$ by its large contribution. From these plots one can conclude unambiguously that the surface state band is responsible for the SP-STM images.

displays a distinctive maximum at an energy of about $\epsilon_{F}$ $-0.4 \mathrm{eV}$. This peak position can be correlated with the bottom of a surface state band which is marked in Fig. 8 by open circles. This surface state was also observed by scanning tunneling spectroscopy measurements as discussed in the previous section (peak $a$ ). Note that due to symmetry arguments this direction remains unchanged when we backfold the band structure from the $p(1 \times 1)$ to the $c(2 \times 2)$ unit cell. Thus, we see a close resemblance of this band with the one discussed in a previous publication (Ref. 35) as responsible for the predicted bias-voltage-dependent corrugation reversal on $\mathrm{W}(110)$. In the band structure of a Mn monolayer on $\mathrm{W}(110)$ we additionally find its exchange-split partner with the bottom of the band at about $\epsilon_{F}-1.2 \mathrm{eV}$ and a very similar dispersion along the displayed high-symmetry line. In this energy regime, the value of the (majority) first star coefficient $\left[n_{\uparrow}^{(1)}(z, \epsilon)\right]$ peak at $\epsilon_{F}-0.4 \mathrm{eV}$ is positive, and according to our sign convention the Mn atom rows with the local magnetization axis along [1 $1 \overline{1} 0]$ should appear as protrusions in the SP-STM image due the (majority) surface state band. The band possesses quite a large dispersion, and still dominates the SP-STM image at the Fermi energy. It is thus also responsible for the experimentally achieved magnetic resolution shown.

However, it cannot directly be concluded from Fig. 8 that the surface state band gives the predominant contribution to the first star coefficient as we have plotted the band structure only in a single direction of the 2D BZ. Therefore, we present in Fig. 16 the contributions to the (majority) first star coefficient from different parts of the two-dimensional Brillouin zone at various energies. From this plot we can clearly see the maximum value at an energy of $\epsilon=\epsilon_{F}-0.4 \mathrm{eV}$ (the large amount of bright area corresponding to a large Brillouin zone integrated first star coefficient) which is located right at the band edge of the surface state about midway along the $\overline{\Gamma M}$ direction. Also the dispersion of this band can be traced, splitting into two branches with increasing energy, one that closes in around $\bar{\Gamma}$ and the other around $\bar{M}$, until both vanish at an energy of about $\epsilon=\epsilon_{F}+0.2 \mathrm{eV}$.

On the other hand, returning to Fig. 8, the second star coefficient $n_{\uparrow}^{(2)}(z, \epsilon)$, with values that are one order of magnitude smaller than the first star coefficient, displays a maximum in the unoccupied states at an energy of $\epsilon_{F}+0.5 \mathrm{eV}$. Therefore, the second star function should contribute to the SP-STM image at positive bias voltages leading to constric- (a)

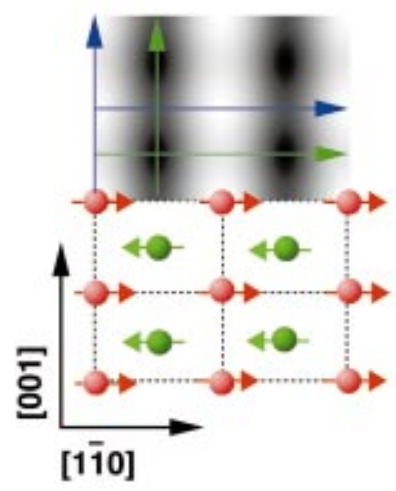

(b)

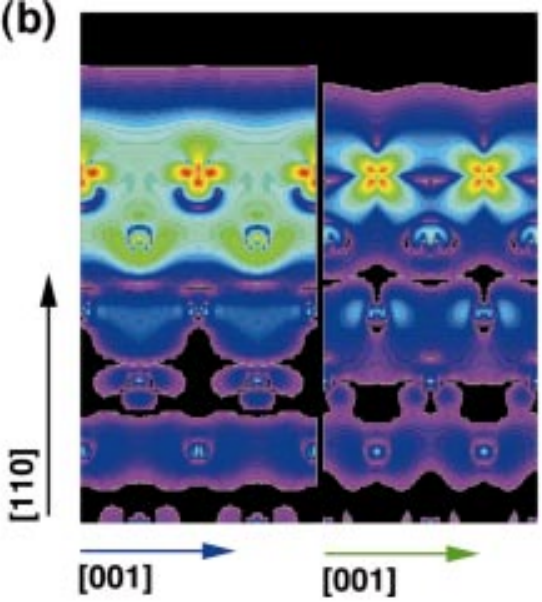

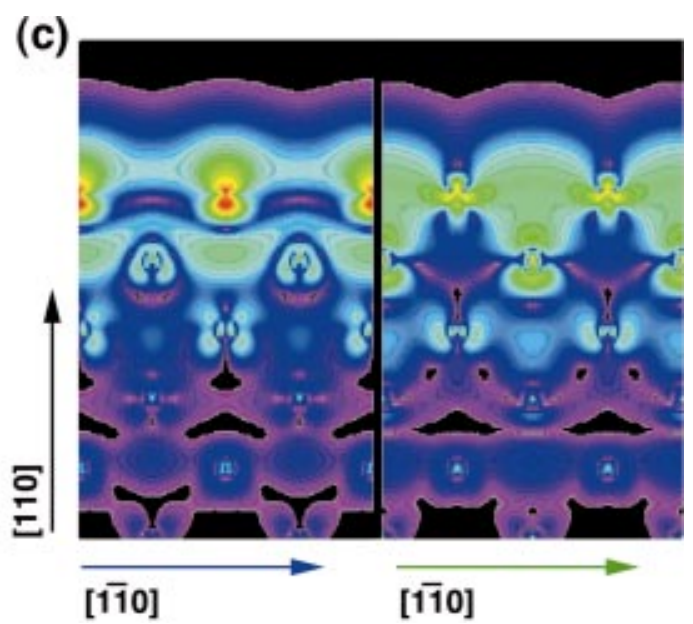

FIG. 17. (Color) Charge-density plot of a single minority spin state of the surface band of the Mn monolayer on W(110) responsible for the SP-STM image. The wave vector is $\mathbf{k}_{\|}=0.68 \overline{\Gamma M}$ and the energy eigenvalue $\epsilon_{\mathbf{k}_{\|} \nu}=\epsilon_{F}-0.11 \mathrm{eV}$. Panel (a) shows the corresponding SP-STM image for a polarization of $P_{T}=0.4$ is shown along with the geometric and magnetic surface structure. (b) and (c) are cross sections along the [001] and [1 $\overline{1} 0]$ directions of the film as indicated by arrows of corresponding color in panel (a). 
tions of the stripes along the [001] direction. At voltages near to the peak position they should become quite pronounced. The correlation of this peak position with states strongly localized at Mn sites as deduced from the band structure is marked by a line at $E-E_{\mathrm{F}} \approx 0.65 \mathrm{eV}$ in Fig. 8 . This very small effect of pronounced constrictions at positive bias voltages has not been verified experimentally although the intensity of the constrictions varied considerably for SP-STM images taken at different tunneling conditions.

The charge density of a single minority spin state $\left(\mathbf{k}_{\|} \nu, \downarrow\right)$ of the surface state band is shown in Fig. 17. Obviously, there is a high localization in the Mn monolayer and additionally in the first layer of the $\mathrm{W}(110)$ substrate. This localization at the two top layers of the whole film is closely related to the surface states present at the pure $\mathrm{W}(110)$ surface. ${ }^{35}$ The orbital character at the $\mathrm{Mn}$ atoms which are imaged as protrusions is $d_{z^{2}}$ while it is $d_{y z}$ for the Mn atoms imaged as depressions. The corresponding SP-STM image is also presented in Fig. 17, which makes the correlation with the cross sections quite easy.

The close relation of the surface state to that of the clean $\mathrm{W}(110)$ surface $^{35}$ hints at the importance of the hybridization at the interface. As the ground state configuration of a $\mathrm{Cr}$ monolayer on $\mathrm{W}(110)$ is also $c(2 \times 2)$ antiferromagnetic, the presence of a similar surface state band is expected. The calculation is actually in accordance with this expectation and since $\mathrm{Cr}$ possesses one electron less than $\mathrm{Mn}$ the surface state band edge shifts closer to the Fermi energy. Correspondingly also the maximum in the corrugation amplitude plot shifts toward zero bias voltage (compare Fig. 15). Thus an even larger corrugation amplitude should be measurable.

\section{SUMMARY AND OUTLOOK}

In conclusion, we have presented a detailed study of the structural, electronic, and magnetic properties of a $\mathrm{Mn}$ monolayer on $\mathrm{W}(110)$ by combining STM experiments with $a b$ initio calculations. From LEED and STM measurements we deduce that the growth mode of $\mathrm{Mn}$ on $\mathrm{W}(110)$ is pseudomorphic for the first and second monolayers. ${ }^{54}$ Further, there is no sign of intermixing at the surface. Taking this geometry into account, ab initio calculations predict a two-dimensional antiferromagnetic superstructure for a $\mathrm{Mn}$ monolayer, i.e., magnetic moments of nearest-neighbor atoms couple antiparallel to each other, and an in-plane easy

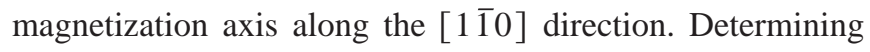
the $\mathrm{Mn}-\mathrm{W}$ interlayer distance from a total-energy calculation results in an interlayer spacing of $2.14 \AA$. At first sight the value seems far from the experimental value of 2.36 $\pm 0.02 \AA$ measured by STM. However, this discrepancy can be readily understood from the large difference in work func- tion of the clean $\mathrm{W}(110)$ surface and a W(110) surface covered by a Mn monolayer, and taking the difference in the electronic structures into account.

The comparison of experimental data for the differential conductivity with the calculated vacuum LDOS averaged over the surface unit cell leads to good agreement for most of the calculated peaks. An occupied surface state at about $\epsilon_{F}$ $-0.4 \mathrm{eV}$ can be clearly identified in the calculation and in the experiment while an experimentally observed peak at $+0.1 \mathrm{eV}$ appears at $-0.06 \mathrm{eV}$ in the calculated spectrum. Further, we find agreement for a peak at a fairly large energy of $\epsilon_{F}+1.7 \mathrm{eV}$. However, a double peak structure at $+0.5 \mathrm{eV}$ and $+0.65 \mathrm{eV}$ cannot be identified in the experimental spectra. We believe that the reason for this discrepancy is the breakdown of the applied Tersoff-Hamann model, which is well justified only at small bias voltages. Thus the agreement for the peak at $\epsilon_{F}+1.7 \mathrm{eV}$ is rather fortuitous.

In order to prove the existence of the calculated $2 \mathrm{D}$ antiferromagnetic ground state of a Mn monolayer on W(110) we computed spin-polarized STM images on the basis of the Tersoff-Hamann model generalized for SP-STM. ${ }^{29}$ General arguments as well as an explicit calculation demonstrate that it becomes possible to observe the magnetic superstructure directly by SP-STM. Thus a direct confirmation of the theoretical predictions is possible and has also been accomplished. The presented SP-STM images unambigiously verify the predicted $2 \mathrm{D}$ antiferromagnetic ground state. Using $\mathrm{Fe}$ - and $\mathrm{Gd}$-coated tungsten tips the calculated in-plane magnetic anisotropy has also been confirmed. A thorough analysis of the calculation further reveals that the occupied surface state band (also identified in the STS measurements) with its energy minimum at about $\epsilon_{F}-0.4 \mathrm{eV}$ is responsible for the magnetic obtained contrast.

In this paper we have established one monolayer of $\mathrm{Mn}$ on W(110) as a model system for two-dimensional itinerant antiferromagnetism. We recommend using this now wellestablished system for further investigations on twodimensional antiferromagnetism, such as, for example, the critical properties, or as a test system for the development of atomically and magnetically resolved atomic force microscopy.

\section{ACKNOWLEDGMENTS}

Two of us (S.H. and S.B.) thank M. Heide for providing the results of the interlayer relaxation of $\mathrm{Mn} / \mathrm{W}(110)$ with LSDA prior to publication. We acknowledge helpful discussions with R. Pfandzelter and financial support from the Deutsche Forschungsgemeinschaft (Grant No. Wi 1277/16-1 and the Graduiertenkolleg "Physik nanostrukturierter Festkörper").

\footnotetext{
*Email address: mbode@physnet.uni-hamburg.de

†Present address: IBM Research Division, T.J. Watson Research Center, Yorktown Heights, 10598 NY.

${ }^{\ddagger}$ Permanent address: Fachbereich Physik, Universität Osnabrück, 49069 Osnabrück, Germany.

${ }^{1}$ F. Bloch, Z. Phys. 61, 206 (1930).
}

\footnotetext{
${ }^{2}$ N.D. Mermin and W. Wagner, Phys. Rev. Lett. 17, 1133 (1966).

${ }^{3}$ For a review, see U. Gradmann, in Handbook of Magnetic Materials, edited by K. H. J. Buschow (Elsevier, Amsterdam, 1993).

${ }^{4}$ R.J. Celotta, D.T. Pierce, G.-C. Wang, S.D. Bader, and G.P. Felcher, Phys. Rev. Lett. 43, 728 (1979).

${ }^{5}$ S.D. Bader, E.R. Moog, and P. Grünberg, J. Magn. Magn. Mater.
} 
53, L295 (1986).

${ }^{6}$ L. Liebermann, J. Clinton, D.M. Edwards, and J. Mathon, Phys. Rev. Lett. 25, 232 (1970).

${ }^{7}$ The apparent discrepancy with the early theoretical considerations (Refs. 1 and 2) is caused by the fact that, in contrast to the assumed isotropic Heisenberg model, every real system exhibits some anisotropy which stabilizes ferromagnetic order.

${ }^{8}$ S. Blügel, M. Weinert, and P.H. Dederichs, Phys. Rev. Lett. 60, 1077 (1988).

${ }^{9}$ S. Blügel and P.H. Dederichs, Europhys. Lett. 9, 597 (1989).

${ }^{10}$ C. Krembel, M.C. Hanf, J.C. Peruchetti, D. Bolmont, and G. Gewinner, J. Magn. Magn. Mater. 93, 529 (1991).

${ }^{11}$ J.E. Ortega and F.J. Himpsel, Phys. Rev. B 47, 16441 (1993).

${ }^{12}$ P. Schieffer, C. Krembel, M.-C. Hanf, M.-H. Tuilier, P. Wetzel, G. Gewinner, and K. Hricovini, Eur. Phys. J. B 8, 165 (1999).

${ }^{13}$ P. Schieffer, C. Krembel, M.-C. Hanf, G. Gewinner, and Y. Gauthier, Phys. Rev. B 62, 2944 (2000).

${ }^{14}$ M. Eder, J. Hafner, and E.G. Moroni, Phys. Rev. B 61, 11492 (2000).

${ }^{15}$ A. Rabe, N. Memmel, A. Steltenpohl, and Th. Fauster, Phys. Rev. Lett. 73, 2728 (1994).

${ }^{16}$ L.Z. Mezey and J. Giber, Jpn. J. Appl. Phys., Part 1 21, 1569 (1982).

${ }^{17}$ G. Rangelov, H.D. Kang, J. Reinmuth, and M. Donath, Phys. Rev. B 61, 549 (2000) and further references therein.

${ }^{18}$ M. Bode, M. Hennefarth, D. Haude, M. Getzlaff, and R. Wiesendanger, Surf. Sci. 432, 8 (1999).

${ }^{19}$ R. Wiesendanger, H.-J. Güntherodt, G. Güntherodt, R.J. Gambino, and R. Ruf, Phys. Rev. Lett. 65, 247 (1990).

${ }^{20}$ R. Wiesendanger, I.V. Shvets, D. Bürgler, G. Tarrach, H.-J. Güntherodt, J.M.D. Coey, and S. Gräser, Science 255, 583 (1992).

${ }^{21}$ S. Heinze, M. Bode, A. Kubetzka, O. Pietzsch, X. Nie, S. Blügel, and R. Wiesendanger, Science 288, 1805 (2000).

${ }^{22}$ U. von Barth and L. Hedin, J. Phys. C 5, 1629 (1972).

${ }^{23}$ J.P. Perdew, J.A. Chevary, S.H. Vosko, K.A. Jackson, M.R. Pederson, D.J. Singh, and C. Fiolhais, Phys. Rev. B 46, 6671 (1992).

${ }^{24}$ V. L. Moruzzi, J. F. Janak, and A. R. Williams, Calculated Electronic Properties of Metals (Pergamon, New York, 1978).

${ }^{25}$ E. Wimmer, H. Krakauer, M. Weinert, and A.J. Freeman, Phys. Rev. B 24, 864 (1981).

${ }^{26}$ M. Weinert, E. Wimmer, and A.J. Freeman, Phys. Rev. B 26, 4571 (1982).

${ }^{27}$ http://www.flapw.de/

${ }^{28}$ X. Qian and W. Hübner, Phys. Rev. B 60, 16192 (1999).

${ }^{29}$ D. Wortmann, S. Heinze, Ph. Kurz, G. Bihlmayer, and S. Blügel, Phys. Rev. Lett. 86, 4132 (2001).

${ }^{30}$ Within the LSDA calculations we have further treated the W $5 p$ and the Mn $3 p$ semicore states as valence states described by local $p$ orbitals added to the LAPW basis set and in a second energy window with flexible energy parameters. The same results were obtained with all approaches.

${ }^{31}$ C. Li, A.J. Freeman, H.J.F. Jansen, and C.L. Fu, Phys. Rev. B 42, 5433 (1990); X. Nie, G. Bihlmayer, and S. Blügel (unpublished).

${ }^{32}$ J. Tersoff and D.R. Hamann, Phys. Rev. Lett. 50, 1998 (1983); Phys. Rev. B 31, 805 (1985).

${ }^{33}$ In this paper the vertical distance $(z)$ is always measured between the center of the surface and tip atom (nucleus-nucleus distance).
${ }^{34}$ We have assumed a collinear magnetic structure of the sample as we find an antiferromagnetic ground state for $\mathrm{Mn} / \mathrm{W}$ (110). The general case of a non collinear structure is treated in Ref. 29.

${ }^{35}$ S. Heinze, S. Blügel, R. Pascal, M. Bode, and R. Wiesendanger, Phys. Rev. B 58, 16432 (1998).

${ }^{36}$ V.T. Binh, S.T. Purcell, N. Garcia, and J. Doglioni, Phys. Rev. Lett. 69, 2527 (1992).

${ }^{37}$ W.A. Hofer, J. Redinger, and P. Varga, Solid State Commun. 113, 245 (1999).

${ }^{38}$ J.A. Stroscio, D.T. Pierce, A. Davies, R.J. Celotta, and M. Weinert, Phys. Rev. Lett. 75, 2960 (1995).

${ }^{39}$ A. Biedermann, O. Genser, W. Hebenstreit, M. Schmid, J. Redinger, R. Podloucky, and P. Varga, Phys. Rev. Lett. 76, 4179 (1996).

${ }^{40}$ M.M.J. Bischoff, C. Konvicka, A.J. Quinn, M. Schmid, J. Redinger, R. Podloucky, P. Varga, and H. van Kempen, Phys. Rev. Lett. 86, 2396 (2001).

${ }^{41}$ B. Voigtländer, V. Scheuch, H.P. Bonzel, S. Heinze, and S. Blügel, Phys. Rev. B 55, 13444 (1997).

${ }^{42}$ S. Heinze, R. Abt, S. Blügel, G. Gilarowski, and H. Niehus, Phys. Rev. Lett. 83, 4808 (1999).

${ }^{43}$ O. Pietzsch, A. Kubetzka, D. Haude, M. Bode, and R. Wiesendanger, Rev. Sci. Instrum. 71, 424 (2000).

${ }^{44}$ M. Bode, R. Pascal, and R. Wiesendanger, Surf. Sci. 344, 185 (1995).

${ }^{45}$ Ch. Witt, U. Mick, M. Bode, and R. Wiesendanger, Rev. Sci. Instrum. 68, 1455 (1997).

${ }^{46} \mathrm{R}$. Wiesendanger, Scanning Probe Microscopy and Spectroscopy (Cambridge University Press, Cambridge, 1994).

${ }^{47}$ Numerical Data and Functional Relationships in Science and Technology, Landolt-Börnstein, New Series, Group III, Vol. 6 (Springer, New York, 1971).

${ }^{48}$ R. Pfandzelter, T. Igel, and H. Winter, Surf. Sci. 389, 317 (1997) and further references therein.

${ }^{49}$ D. Tian, S.C. Wu, F. Jona, and P.M. Marcus, Solid State Commun. 70, 199 (1989).

${ }^{50} \mathrm{We}$ would like to note that a wrong value of the monatomic step height on a W (110) surface was used for calibration in a previous publication (Ref. 18).

${ }^{51}$ U. Gradmann and G. Waller, Surf. Sci. 116, 539 (1982).

${ }^{52}$ H. Bethge, D. Heuer, Ch. Jensen, K. Reshöft, and U. Köhler, Surf. Sci. 331-333, 878 (1995).

${ }^{53}$ C. Jensen, K. Reshöft, and U. Köhler, Appl. Phys. A: Mater. Sci. Process. 62, 217 (1996).

${ }^{54}$ In fact, we showed in a previous publication (Ref. 18) that the third atomic Mn layer grows pseudomorphically, too.

${ }^{55}$ C.G. Shull and M.K. Wilkinson, Rev. Mod. Phys. 25, 100 (1953).

${ }^{56}$ J.S. Kasper and B.W. Roberts, Phys. Rev. 101, 537 (1956).

${ }^{57}$ M. Heide, G. Bihlmayer, and S. Blügel (unpublished).

${ }^{58}$ H. Ohnishi, Y. Kondo, and K. Takayanagi, Surf. Sci. 415, L1061 (1998).

${ }^{59}$ The deficiency of the Tersoff-Hamann model in explaining STM images of close-packed metal surfaces qualitatively has been related to special electronic states of the tip or slight deformations of the tip due to the close proximity to the surface. However, it has also been shown theoretically (Ref. 32) and experimentally (Ref. 60) that with increasing size of the unit cell, e.g., due to a chemical or-as in our case-magnetic superstructure, the corrugation becomes independent of particular tip states. 
Good quantitative agreement is thus achieved for the spinpolarized measurements as well for earlier studies of surface metal alloys (Ref. 42).

${ }^{60}$ J.V. Barth, H. Brune, G. Ertl, and R.J. Behm, Phys. Rev. B 42, 9307 (1990).

${ }^{61}$ M. Bode, M. Getzlaff, and R. Wiesendanger, Phys. Rev. Lett. 81, 4256 (1998).

${ }^{62}$ Since we are currently not able to control the azimuthal angle of the tip magnetization in our experiments we cannot decide experimentally which in-plane direction, i.e., the [001] or the [1 $1 \overline{1} 0]$ direction, is the easy magnetization direction of a $\mathrm{Mn}$ monolayer on W (110).

${ }^{63}$ M. Bode, M. Getzlaff, and R. Wiesendanger, J. Vac. Sci. Technol. A 17, 2228 (1999).

${ }^{64}$ O. Pietzsch, A. Kubetzka, M. Bode, and R. Wiesendanger, Phys. Rev. Lett. 84, 5212 (2000). 